\title{
Locally Restricted Compositions I. Restricted Adjacent Differences
}

\author{
Edward A. Bender \\ Department of Mathematics \\ University of California, San Diego \\ La Jolla, CA 92093-0112 \\ ebender@ucsd.edu \\ E. Rodney Canfield \\ Department of Computer Science \\ University of Georgia \\ Athens, GA 30602 \\ erc@cs . uga.edu
}

AMS Subject Classification: 05A15, 05A16

Submitted: May 28, 2005; Accepted: Oct 31, 2005; Published: Nov 7, 2005

\begin{abstract}
We study compositions of the integer $n$ in which the first part, successive differences, and the last part are constrained to lie in prescribed sets $\mathcal{L}, \mathcal{D}, \mathcal{R}$, respectively. A simple condition on $\mathcal{D}$ guarantees that the generating function $f(x, \mathcal{L}, \mathcal{D}, \mathcal{R})$ has only a simple pole on its circle of convergence and this at $r(\mathcal{D})$, a function independent of $\mathcal{L}$ and $\mathcal{R}$. Thus the number of compositions is asymptotic to $\operatorname{Ar}(\mathcal{D})^{-n}$ for a suitable constant $A=A(\mathcal{L}, \mathcal{D}, \mathcal{R})$. We prove a multivariate central and local limit theorem and apply it to various statistics of random locally restricted compositions of $n$, such as number of parts, numbers of parts of given sizes, and number of rises. The first and last parts are shown to have limiting distributions and to be asymptotically independent. If $\mathcal{D}$ has only finitely many positive elements $\mathcal{D}^{+}$, or finitely many negative elements $\mathcal{D}^{-}$, then the largest part and number of distinct part sizes are almost surely $\Theta\left((\log n)^{1 / 2}\right)$. On the other hand, when both $\mathcal{D}^{+}$and $\mathcal{D}^{-}$ have a positive asymptotic lower "local log-density", we prove that the largest part and number of distinct part sizes are almost surely $\Theta(\log n)$, and we give sufficient conditions for the largest part to be almost surely asymptotic to $\log _{1 / r(\mathcal{D})} n$.
\end{abstract}




\section{Introduction}

Let $\mathbb{Z}$ be the integers and $\mathbb{N}$ the strictly positive integers. When we refer to either positive or negative numbers, we exclude zero.

Definition 1 (( $\mathcal{L}, \mathcal{D}, \mathcal{R})$ compositions) Let $\mathcal{L} \subseteq \mathbb{N}$ and $\mathcal{R} \subseteq \mathbb{N}$ be nonempty. Let $\mathcal{D} \subseteq \mathbb{Z}$ contain at least one positive integer and at least one negative integer. For positive integers $n$ and $k$, an $(\mathcal{L}, \mathcal{D}, \mathcal{R})$ composition of $n$ into $k$ parts is an ordered $k$-tuple of positive integers $a_{1}, a_{2}, \ldots, a_{k}$ satisfying

(a) $\sum_{i=1}^{k} a_{i}=n$,

(b) $a_{1} \in \mathcal{L}, a_{k} \in \mathcal{R}$, and

(c) $a_{i+1}-a_{i} \in \mathcal{D}, 1 \leq i<k$.

If there are no $(\mathcal{L}, \mathcal{D}, \mathcal{R})$ compositions, we say $(\mathcal{L}, \mathcal{D}, \mathcal{R})$ is trivial. Note that 0 has no compositions.

Remark 1 Condition (a) is the standard definition of a composition of the positive integer $n$. We impose the additional restrictions that the first part, successive differences, and last part be in prescribed sets.

Remark 2 If the elements of $\mathcal{D}$ were allowed to be all of one sign, the parts of the composition would be monotonic and so we would be dealing with partitions of numbers, which behave very differently from our compositions.

Lemma $1(\mathcal{L}, \mathcal{D}, \mathcal{R})$ is nontrivial if and only if there exist $\ell \in \mathcal{L}$ and $r \in \mathcal{R}$ such that $\operatorname{gcd}(\mathcal{D})$ divides $r-\ell$.

Proof: If $a_{1}, \ldots, a_{k}$ is an $(\mathcal{L}, \mathcal{D}, \mathcal{R})$ composition, we have $d_{i}=a_{i}-a_{i-1} \in \mathcal{D}$ and so $a_{k}-a_{1}=d_{2}+\cdots+d_{k}$. Consequently $\operatorname{gcd}(\mathcal{D})$ divides $a_{k}-a_{1}$. Conversely, suppose $\ell \in \mathcal{L}$, $r \in \mathcal{R}$ and $\operatorname{gcd}(\mathcal{D}) \mid(r-\ell)$. If $r-\ell=0$, then $\ell$ is an $(\mathcal{L}, \mathcal{D}, \mathcal{R})$ composition, If $r-\ell \neq 0$, we claim we can write

$$
r-\ell=d_{2}+\cdots+\cdots+d_{k} \text { for some } d_{i} \in \mathcal{D} \text { with } d_{i}>0 \text { if and only if } i \leq j .
$$

(That is, the positive differences $d_{i}$ precede the negative.) Let $a_{1}=\ell$ and $a_{i}=a_{i-1}+d_{i}$ for $2 \leq i \leq k$. Since the $a_{i}$ increase and then decrease and since $a_{k}=r>0$, the $a_{i}$ are all positive.

It remains to prove the claim. By assumption $r-\ell=\sum m_{i} d_{i}$ for some $m_{i} \in \mathbb{Z}$. If $m_{i}<0$, choose $d \in \mathcal{D}$ with the opposite sign from $d_{i}$ and let $M \geq\left|m_{i}\right|$ be a multiple of $|d|$. Replace the term $m_{i} d_{i}$ with the two terms $\left(-d_{i} M / d\right) d$ and $\left(M+m_{i}\right) d_{i}$, noting that $-d_{i} M / d>0$ because $d_{i}$ and $d$ are of opposite sign. Once all coefficients $m_{i}$ are positive, they can all be made to equal +1 by repetition of $d$ 's; then the $d$ 's may be permuted to place positive differences first. 
Definition 2 (counts and generating functions) Let $c_{\mathcal{L}, \mathcal{D}, \mathcal{R}}(n, k)$ be the number of $(\mathcal{L}, \mathcal{D}, \mathcal{R})$ compositions of $n$ having exactly $k$ parts. Define the power series $f(x, t, \mathcal{L}, \mathcal{D}, \mathcal{R})$ by

$$
f(x, t, \mathcal{L}, \mathcal{D}, \mathcal{R}):=\sum_{n, k} c_{\mathcal{L}, \mathcal{D}, \mathcal{R}}(n, k) x^{n} t^{k} .
$$

Since $\mathcal{D}$ is often fixed, we usually omit it, and sometimes we omit $\mathcal{L}$ and $\mathcal{R}$ when the meaning is clear. Let $c(n)=\sum_{k} c(n, k)$. Thus $\sum_{n} c(n) x^{n}=f(x, 1)$. We may introduce additional variables to keep track of additional counts.

Example 1 (ordinary compositions) When $\mathcal{L}=\mathcal{R}=\mathbb{N}=\{1,2, \ldots\}$, and $\mathcal{D}=\mathbb{Z}$, we have ordinary compositions. Since $c(n, k)=\left(\begin{array}{c}n-1 \\ k-1\end{array}\right)$, the number of such compositions, the distribution of the number of parts, and the distribution of the end parts is easily studied. With $z_{k}$ keeping track of parts of size $k$, the generating function for a single part is $t \sum x^{k} z_{k}$ and so the generating function for the compositions is $\left(1-t \sum x^{k} z_{k}\right)^{-1}$, which can be used to obtain information such as a multivariate normal. More difficult are the largest part and number of distinct parts. It is known that the latter two are almost surely asymptotic to $\log _{2} n$. Information about their distributions is more difficult to obtain. See Hitczenko and Stengle [8] and Hwang and Yeh [9]. A historical discussion and various results are given by Hitczenko and Louchard [7].

Example 2 (Carlitz compositions) When $\mathcal{L}=\mathcal{R}=\mathbb{N}$ and $\mathcal{D}$ is the nonzero integers, we have Carlitz compositions: compositions where adjacent parts must be different. Carlitz [4] obtained the formula

$$
f(x, t, \mathcal{L}, \mathcal{R})=\frac{g(x, t)}{1-g(x, t)} \quad \text { where } \quad g(x, t)=\sum_{k \geq 1} \frac{(-1)^{k-1}(x t)^{k}}{1-x^{k}} .
$$

This can be analyzed to establish various facts, including:

- the radius of convergence of $f(x, 1, \mathcal{L}, \mathcal{R})$ is $r \approx 0.78397$,

- $x=r$ is a simple pole and the only singularity on the circle of convergence,

- the number of parts in a randomly chosen Carlitz composition is asymptotically normal with mean and variance asymptotically proportional to $n$.

With further work, it can be shown that the largest part and number of distinct parts are almost surely asymptotic to $\log _{1 / r}(n)$. See the papers $[5,7,10,11]$ for further discussion, additional results, and more precise asymptotics. Various people have studied rises and falls in Carlitz and other compositions. See Heubach and Mansour [6] for results and further discussion.

Example 3 (ballot-like compositions) When $\mathcal{L}=\mathcal{R}=\{1\}$ and $\mathcal{D}=\{ \pm 1\}$, the situation is closely related to elections that end in a tie although the first candidate always leads in votes until the end. The election viewpoint focuses on what happens when $k$ (the 
number of votes) is fixed and $n$ varies. There is a fairly extensive literature on this in probability theory. For example, the largest part is $\Theta\left(k^{1 / 2}\right)[12]$. The composition viewpoint focuses on what happens when $n$ is fixed. In this case, we will see that $k$ is expected to grow linearly with $n$ and the largest part is expected to be $\Theta\left((\log n)^{1 / 2}\right)$. We are unaware of literature from this viewpoint.

For sets $\mathcal{P}$ and $\mathcal{S}$ of integers define

$$
\begin{aligned}
\mathcal{P}^{+} & :=\{p>0 \mid p \in \mathcal{P}\} & & \text { (positive elements) } \\
\mathcal{P}^{-} & :=\{p>0 \mid-p \in \mathcal{P}\} & & \text { (unsigned negative elements) } \\
\mathcal{P} \pm \mathcal{S} & :=\{p \pm s \mid p \in \mathcal{P} \text { and } s \in \mathcal{S}\} & & \text { (sum or difference of elements). }
\end{aligned}
$$

The following theorem summarizes our major results.

Theorem 1 (main theorem) Suppose either $\operatorname{gcd}\left(\mathcal{D}^{+} \cap \mathcal{D}^{-}\right)=1$ or $\operatorname{gcd}(\mathcal{D}-\mathcal{D})=1$. In what follows, random variables are based on the uniform distribution on $(\mathcal{L}, \mathcal{D}, \mathcal{R})$ compositions whose parts sum to $n$ and limits are as $n \rightarrow \infty$.

(a) There are nonzero constants $A(\mathcal{L}, \mathcal{D}, \mathcal{R})$ and $r(\mathcal{D})$ such that

$$
c_{\mathcal{L}, \mathcal{R}}(n) \sim A r^{-n}
$$

(A and $r$ can be computed by applying Theorem 3 to (3.2).)

(b) Let $P_{n}$ be the number of parts in a composition. There is a central limit theorem independent of $\mathcal{L}$ and $\mathcal{R}$; that is, there are constants $\mu(\mathcal{D})>0$ and $\sigma(\mathcal{D})>0$ such that

$$
\operatorname{Pr}\left(P_{n}<x\right)=\frac{1}{\sqrt{2 \pi n} \sigma} \int_{-\infty}^{x} e^{-(t-n \mu) / 2 n \sigma^{2}} d t+o(1)
$$

uniformly in $x$.

If we have $\operatorname{gcd}(\mathcal{D}-\mathcal{D})=1$, then there is a local limit theorem:

$$
\operatorname{Pr}\left(P_{n}=k\right)=\frac{e^{-(k-\mu n)^{2} / 2 n \sigma^{2}}+o(1)}{\sqrt{2 \pi n} \sigma}
$$

uniformly in $k$.

(c) In "nondegenerate" cases the joint distribution for number of rises, falls, equalities (when $0 \in \mathcal{D}$ ), local maxima and minima, various part sizes, and various adjacent differences is asymptotically normal with means vector and covariance matrix proportional to $n$. The meaning of "nondegenerate" and a way to test for a local limit theorem is discussed in the proof of (c) in Section 5.

(d) Let $F_{n}$ and $L_{n}$ be the first and last parts of a composition. All moments of $F_{n}$ and of $L_{n}$ about 0 are finite and they have limiting distributions which are independent. 
(e) Let $M_{n}$ be the largest part in a composition of $n$. If $g(n) \rightarrow \infty$, then

$$
\operatorname{Pr}\left(M_{n}<\log _{1 / r} n+g(n)\right) \rightarrow 1,
$$

where $r$ is as in (a).

(f) Let $D_{n}$ be the number of different part sizes in a composition of $n$. If $\mathcal{D}^{+}$or $\mathcal{D}^{-}$is finite, then $D_{n}=\Theta\left((\log n)^{1 / 2}\right)$ almost surely and $M_{n}=\Theta\left((\log n)^{1 / 2}\right)$ almost surely.

(g) Suppose $\mathcal{D}^{+}$and $\mathcal{D}^{-}$are both infinite. If the elements of $\mathcal{D}^{+}$(respectively $\mathcal{D}^{-}$or $\mathcal{D}^{+} \cap \mathcal{D}^{-}$) are $d_{1}<d_{2}<\cdots$, define $\rho^{+}$(respectively $\rho^{-}$or $\rho$ ) to be $\lim \inf _{i \rightarrow \infty} d_{i-1} / d_{i}$. If $\rho^{-}>0$ and $\rho^{+}>0$, then:

(i) $D_{n}=\Theta(\log n)$ almost surely;

(ii) for all $\delta>0$

$$
\operatorname{Pr}\left(M_{n}>(B-\delta) \log _{1 / r} n\right) \rightarrow 1,
$$

where $B=\max \left(\rho^{+} \rho^{-}, \rho\right)$;

(iii) if $\rho^{+}=\rho^{-}=1$, then $D_{n} \sim M_{n} \sim \log _{1 / r} n$ almost surely.

Remark 3 If $0 \in \mathcal{D}$, then $\operatorname{gcd}(\mathcal{D}-\mathcal{D})=\operatorname{gcd}(\mathcal{D}) \leq \operatorname{gcd}\left(\mathcal{D}^{+} \cap \mathcal{D}^{-}\right)$and so the $\operatorname{gcd}$ conditions in the theorem can all be replaced with $\operatorname{gcd}(\mathcal{D})=1$.

Remark 4 We conjecture that the result $D_{n} \sim M_{n}$ in (g.iii) is true if $\operatorname{gcd}(\mathcal{D})=1$ with no additional conditions on $\mathcal{D}$. Of greater interest is $M_{n}-D_{n}$. The distributions of $M_{n}$ and $D_{n}$ have been studied for ordinary and Carlitz compositions, from which it follows that the expected value of $M_{n}-D_{n}$ is nearly constant. This may be true under the much weaker condition $\operatorname{gcd}(\mathcal{D})=1$.

If $(\mathcal{L}, \mathcal{D}, \mathcal{R})$ is nontrivial but $\operatorname{gcd}(\mathcal{D})>1$, the situation is more complicated than in Theorem 1. For example, if $\mathcal{L}=\mathcal{R}=\{d\}$ and $\mathcal{D}=\{ \pm d\}$, then the sum of the parts in an $(\mathcal{L}, \mathcal{D}, \mathcal{R})$ composition is a multiple of $d$. It is also possible for (a) to hold but for $r$ to depend on $\mathcal{L}$ and $\mathcal{R}$ as well as $\mathcal{D}$. As an example, we will prove

Theorem 2 (unequal radii) Let $\mathcal{L}_{i}=\mathcal{R}_{i}=\{i\}$. If $\mathcal{D}^{+}=\mathcal{D}^{-}$and $\operatorname{gcd}(\mathcal{D})=\delta$ is an odd prime, then each of the generating functions $f\left(x, 1, \mathcal{L}_{i}, \mathcal{R}_{i}\right)(1 \leq i<\delta)$ has a unique singularity on its circle of convergence and the singularity is a simple pole. Thus $c_{\mathcal{L}_{i}, \mathcal{R}_{i}}(n) \sim A_{i} r_{i}^{-n}$ in each case; however, $r_{1}<r_{2}<\cdots<r_{\delta-1}$.

In Section 2 we prove a central and local limit theorem for functions of a particular form. Sections 3-8 are devoted to proving Theorems 1 and 2. Section 9 discusses the average number of parts of size $k$ for fixed $k$. The final section presents some computational results.

In [2] we prove a local limit theorem for a more general class of locally restricted compositions: the restrictions may involve nonadjacent parts, and can be more general 
than what is expressable in terms of differences. That result implies the local part of Theorem 1(c), but, unlike the proof of Theorem 1, does not provide a practical way to estimate the parameters accurately.

\section{A central and local limit theorem}

We shall prove Theorem 1 by using the following theorem, which may be of some independent interest.

Theorem 3 (a limit theorem) Suppose that $\mathbf{t}$ keeps track of $\ell$ parameters and that

$$
A(x, \mathbf{t})=F(x, \mathbf{t})+\frac{G(x, \mathbf{t})}{1-H(x, \mathbf{t})}
$$

where $A, F, G$ and $H$ have multivariate power series expansions about the origin and the coefficients of $A$ and $H$ are nonnegative. Define

$$
\mathcal{H}_{n}:=\left\{(n, \mathbf{k}) \mid h_{n, \mathbf{k}} \neq 0\right\} \subseteq \mathbb{Z}^{\ell+1} \quad \text { and } \quad \mathcal{H}:=\bigcup_{n \geq 1} \mathcal{H}_{n}
$$

where $H(x, \mathbf{t})=\sum h_{n, \mathbf{k}} x^{n} \mathbf{t}^{\mathbf{k}}$ and $\mathbf{t}^{\mathbf{k}}=t_{1}^{k_{1}} \cdots t_{\ell}^{k_{\ell}}$. Suppose that there are $\rho>r>0$ and $\tau>1$ such that

(a) the series for $F, G$ and $H$ converge whenever $|x|<\rho$ and $\left|t_{i}\right|<\tau$ for $1 \leq i \leq \ell$,

(b) $H(r, \mathbf{1})=1$,

(c) $G(r, \mathbf{1}) \neq 0$,

(d) $h_{0, \mathbf{k}}=0$ for all $\mathbf{k}$,

(e) $\operatorname{gcd}\left\{n \mid \mathcal{H}_{n} \neq \emptyset\right\}=1$.

Then

$$
a_{n}:=\left[x^{n}\right] A(x, \mathbf{1}) \sim \frac{G(r, \mathbf{1})}{r H_{x}(r, \mathbf{1})} r^{-n} .
$$

Let $X_{n}$ be a random variable with

$$
\operatorname{Pr}\left(X_{n}=\mathbf{k}\right)=\frac{\left[x^{n} \mathbf{t}^{\mathbf{k}}\right] A(x, \mathbf{t})}{\left[x^{n}\right] A(x, \mathbf{1})} .
$$

If, in addition to the previous conditions,

(f) $\mathcal{H}$ spans $\mathbb{R}^{\ell+1}$ over $\mathbb{R}$, 
then we have a central limit theorem for $X_{n}$ : the distribution of $X_{n}$ is asymptotically normal with mean $n \mathbf{m}$ and covariance matrix $n B$ where

$$
m_{i}=\frac{H_{t_{i}}(r, \mathbf{1})}{r H_{x}(r, \mathbf{1})}>0 \quad \text { and } \quad B_{i, j}=\frac{\sum_{n, \mathbf{k}}\left(m_{i} n-k_{i}\right)\left(m_{j} n-k_{j}\right) h_{n, \mathbf{k}} r^{n}}{r H_{x}(r, \mathbf{1})} .
$$

If instead of $(f)$ we have the stronger condition

(g) $\mathcal{H}$ spans $\mathbb{Z}^{\ell+1}$ over $\mathbb{Z}$

then we have a local limit theorem for $X_{n}$ :

$$
\operatorname{Pr}\left(X_{n}=\mathbf{k}\right)=\frac{\exp \left(-(\mathbf{k}-n \mathbf{m})^{\mathrm{t}}(2 n B)^{-1}(\mathbf{k}-n \mathbf{m})\right)+o(1)}{\sqrt{\operatorname{det}(2 \pi n B)}}
$$

uniformly in $\mathbf{k}$.

Proof: Condition (a) guarantees that the singularities of $A(x, \mathbf{t})$ with $|x|<\rho$ and $\left|t_{i}\right|<\tau$ are due to $H(x, \mathbf{t})=1$. Furthermore (b) and (c) guarantee that there is a singularity of $A(x, \mathbf{1})$ at $x=r$. Thus the singularities of $A(x, 1)$ with $|x| \leq r$ are given by the solutions to $H(x, 1)=1$. Since $H$ has nonnegative coefficients and some $\mathcal{H}_{n}$ is nonempty, $x=r$ is a root of multiplicity 1 of $H(x, \mathbf{1})=1$. Since $G(r, \mathbf{1}) \neq 0, x=r$ is a simple pole of $A(x, \mathbf{1})$. Condition (e) and the nonnegativity of the coefficients of $H(r, \mathbf{1})$ insure that this simple pole is the only root of $H(x, \mathbf{1})=1$ with $|x| \leq r$ and hence the only singularity of $A(r, \mathbf{1})$ on its circle of convergence. Equation (2.1) follows.

We now prove the central limit theorem. For $\mathbf{t} \in \mathbb{R}^{\ell}$ and $\mathbf{t}$ near $\mathbf{1}$, let $r(\mathbf{t})$ be the radius of convergence of $H(x, \mathbf{t})$. Thus $r(\mathbf{1})=r$. Since $h_{n, \mathbf{k}} \geq 0$, it follows from (f) that $H_{x}(r, \mathbf{1})>0$ and so $r(\mathbf{t})$ is analytic in a neighborhood of $\mathbf{t}=\mathbf{1}$. Corollary 1 of [3] gives a central limit result with

$$
m_{i}=\left.\frac{-\partial \log r(\mathbf{t})}{\partial \log t_{i}}\right|_{\mathbf{t}=\mathbf{1}}=\left.\frac{-t_{i}}{r(\mathbf{t})} \frac{\partial r(\mathbf{t})}{\partial t_{i}}\right|_{\mathbf{t}=\mathbf{1}}=\left.\frac{-1}{r} \frac{\partial r(\mathbf{t})}{\partial t_{i}}\right|_{\mathbf{t}=\mathbf{1}}
$$

and

$$
\begin{aligned}
B_{i, j} & =\left.\frac{-\partial^{2} \log r(\mathbf{t})}{\partial \log t_{i} \partial \log t_{j}}\right|_{\mathbf{t}=\mathbf{1}}=\left.\frac{t_{j} \partial}{\partial t_{j}}\left(\frac{-t_{i}}{r(\mathbf{t})} \frac{\partial r(\mathbf{t})}{\partial t_{i}}\right)\right|_{\mathbf{t}=\mathbf{1}} \\
& =\left.\frac{-\delta_{i, j}}{r} \frac{\partial r(\mathbf{t})}{\partial t_{i}}\right|_{\mathbf{t}=\mathbf{1}}+\left.\frac{1}{r^{2}} \frac{\partial r(\mathbf{t})}{\partial t_{j}} \frac{\partial r(\mathbf{t})}{\partial t_{i}}\right|_{\mathbf{t}=\mathbf{1}}-\left.\frac{1}{r} \frac{\partial^{2} r(\mathbf{t})}{\partial t_{i} \partial t_{j}}\right|_{\mathbf{t}=\mathbf{1}} \\
& =\delta_{i, j} m_{i}+m_{i} m_{j}-\left.\frac{1}{r} \frac{\partial^{2} r(\mathbf{t})}{\partial t_{i} \partial t_{j}}\right|_{\mathbf{t}=\mathbf{1}},
\end{aligned}
$$

provided the $m_{i}$ are positive and $B$ is positive definite. We begin by showing that (2.2) is correct (which implies $m_{i}>0$ ) and then prove $B$ is positive definite. From now on, it is understood that unspecified evaluations are at $(x, \mathbf{t})=(r, \mathbf{1})$. 
Since $r(\mathbf{t})$ is given by $H(r(\mathbf{t}), \mathbf{t})=1$, we have

$$
\begin{aligned}
\frac{d H}{d t_{i}} & =0=H_{x} \frac{\partial r}{\partial t_{i}}+H_{t_{i}} \\
\frac{d^{2} H}{d t_{i} d t_{j}} & =0=\left(H_{x, x} \frac{\partial r}{\partial t_{i}} \frac{\partial r}{\partial t_{j}}+H_{x, t_{j}} \frac{\partial r}{\partial t_{i}}+H_{x} \frac{\partial^{2} r}{\partial t_{i} \partial t_{j}}\right)+\left(H_{t_{i}, x} \frac{\partial r}{\partial t_{j}}+H_{t_{i}, t_{j}}\right) .
\end{aligned}
$$

It follows that $m_{i}=-(1 / r)\left(\partial r / \partial t_{i}\right)=H_{t_{i}} / r H_{x}$ and so the value of $m_{i}$ in $(2.2)$ is correct.

We now show that the value of $B_{i, j}$ is correct. From (2.4) and $m_{i}=-(1 / r)\left(\partial r / \partial t_{i}\right)$,

$$
-\frac{1}{r} \frac{\partial^{2} r}{\partial t_{i} \partial t_{j}}=\frac{1}{r H_{x}}\left(r^{2} H_{x, x} m_{i} m_{j}-r H_{x, t_{i}} m_{j}-r H_{x, t_{j}} m_{i}+H_{t_{i}, t_{j}}\right)
$$

and so

$$
r H_{x} B_{i, j}=\delta_{i, j} r H_{x} m_{i}+r H_{x} m_{i} m_{j}+\left(r^{2} H_{x, x} m_{i} m_{j}-r H_{x, t_{i}} m_{j}-r H_{x, t_{j}} m_{i}+H_{t_{i}, t_{j}}\right)
$$

Note that

$$
\begin{aligned}
r H_{x} & =\sum n h_{n, \mathbf{k}} r^{n} \\
H_{t_{i}} & =\sum k_{i} h_{n, \mathbf{k}} r^{n} \\
r^{2} H_{x, x} & =\sum\left(n^{2}-n\right) h_{n, \mathbf{k}} r^{n} \\
r H_{x, t_{i}} & =\sum n k_{i} h_{n, \mathbf{k}} r^{n} \\
H_{t_{i}, t_{j}} & =\sum k_{i} k_{j} h_{n, \mathbf{k}} r^{n}-\delta_{i, j} \sum k_{i} h_{n, \mathbf{k}} r^{n},
\end{aligned}
$$

where the sums are over $n$ and $\mathbf{k}$. Putting everything together,

$$
\begin{aligned}
r H_{x} B_{i, j}= & \sum\left(\delta_{i, j} n m_{i}+n m_{i} m_{j}\right. \\
& \left.\quad+\left(n^{2}-n\right) m_{i} m_{j}-n k_{i} m_{j}-n k_{j} m_{i}+k_{i} k_{j}-\delta_{i, j} k_{i}\right) h_{n, \mathbf{k}} r^{n} \\
= & \sum\left(\left(n m_{i}-k_{i}\right)\left(n m_{j}-k_{j}\right)+\delta_{i, j}\left(n m_{i}-k_{i}\right)\right) h_{n, \mathbf{k}} r^{n} .
\end{aligned}
$$

From the formula for $m_{i}$,

$$
0=r H_{x} m_{i}-H_{t_{i}}=\sum\left(n m_{i}-k_{i}\right) h_{n, \mathbf{k}} r^{n} .
$$

Thus the formula for $B_{i, j}$ in (2.2) is correct.

Since $r H_{x}>0, B$ is positive definite if $\mathbf{v}^{\mathrm{t}}\left(r H_{x} B\right) \mathbf{v}>0$ for all nonzero $\mathbf{v} \in \mathbb{R}^{\ell}$. We have

$$
\mathbf{v}^{\mathrm{t}}\left(r H_{x} B\right) \mathbf{v}=\sum_{n, \mathbf{k}}\left(\sum_{i}\left(n m_{i}-k_{i}\right) v_{i}\right)^{2} h_{n, \mathbf{k}} r^{n}=\sum_{n, \mathbf{k}}\left((n \mathbf{m}-\mathbf{k})^{\mathrm{t}} \mathbf{v}\right)^{2} h_{n, \mathbf{k}} r^{n}
$$


Thus it suffices to show that the set of equations

$$
\left\{(n \mathbf{m}-\mathbf{k})^{\mathrm{t}} \mathbf{v}=0 \mid h_{n, \mathbf{k}} \neq 0\right\} \quad \text { has only the trivial solution. }
$$

This will be true if $\mathcal{S}=\left\{n \mathbf{m}-\mathbf{k} \mid h_{n, \mathbf{k}} \neq 0\right\}$ spans $\mathbb{R}^{\ell}$ over $\mathbb{R}$. Let $\mathbf{w} \in \mathbb{R}^{\ell}$. By (f), $\operatorname{span} \mathcal{H}=\mathbb{R}^{\ell+1}$. Thus $(0,-\mathbf{w})=\sum r_{i}\left(n_{i}, \mathbf{k}_{i}\right)$ for some $r_{i} \in \mathbb{R}$ and $\left(n_{i}, \mathbf{k}_{i}\right) \in \mathcal{H}$. Thus $0=\sum r_{i} n_{i}$ and $\mathbf{w}=-\sum r_{i} \mathbf{k}_{i}$. Consequently $\mathbf{w}=\sum r_{i}\left(n_{i} \mathbf{m}-\mathbf{k}_{i}\right) \in \operatorname{span} \mathcal{S}$ and so $\mathcal{S}$ spans $\mathbb{R}^{\ell}$. This completes the proof of the central limit theorem.

By Corollary 2 of [3], the local limit theorem will follow if $H(x, \mathbf{t})=1$ has no solutions with $|x|=r$ and $\left|t_{i}\right|=1$ for $1 \leq i \leq \ell$ other than $x=r$ and $\mathbf{t}=\mathbf{1}$. Note that

$$
\left|\sum h_{n \mathbf{k}} x^{n} \mathbf{t}^{\mathbf{k}}\right| \leq \sum h_{n, \mathbf{k}} r^{n}=1
$$

with equality if and only if $\arg \left(x^{n} \mathbf{t}^{\mathbf{k}}\right)$ is constant for all $(n, \mathbf{k})$ where $h_{n, \mathbf{k}} \neq 0$. Since we want the sum to equal 1 in value, not just magnitude, it follows that, when $|x|=r$ and $\left|t_{i}\right|=1$,

$$
H(x, \mathbf{t})=1 \text { if and only if } \arg \left(x^{n} \mathbf{t}^{\mathbf{k}}\right)=0 \text { whenever } h_{n, \mathbf{k}} \neq 0 .
$$

Since $\mathcal{H}$ spans $\mathbb{Z}^{\ell+1}$ over $\mathbb{Z}$, we have $\arg (x)=\arg \left(t_{i}\right)=0$. This completes the proof.

\section{Some functional equations}

We discuss in detail recursions that keep track of the sum and number of parts; that is, recursions for the $f$ of Definition 2. Then we briefly sketch the modifications needed to keep track of some other quantities. The important fact about these modifications is not the details but rather the observation, to be made later, that Theorem 3 can be applied to obtain a joint normal distribution.

\subsection{Keeping track of number of parts}

For any set $\mathcal{S}$ of positive integers define

$$
\mathcal{S}^{\prime}:=\{s-1 \mid s \in \mathcal{S} \text { and } s>1\}
$$

Define

$$
\chi(\text { statement }):= \begin{cases}1, & \text { if statement is true } \\ 0, & \text { if statement is false. }\end{cases}
$$

We will prove

Theorem 4 (the recursion) Let $\mathcal{L}, \mathcal{D}, \mathcal{R}$ be as in Definition 1 , define $\mathcal{D}^{+}$and $\mathcal{D}^{-}$by (1.2) and define $\mathcal{L}^{\prime}, \mathcal{R}^{\prime}$ by (3.1). Then

$$
\begin{aligned}
f(x, t, \mathcal{L}, \mathcal{R}) & =f\left(x, x t, \mathcal{L}^{\prime}, \mathcal{R}^{\prime}\right) \\
& +\frac{\left(f\left(x, x t, \mathcal{L}^{\prime}, \mathcal{D}^{-}\right)+\chi(1 \in \mathcal{L})\right) z(x t)\left(f\left(x, x t, \mathcal{D}^{+}, \mathcal{R}^{\prime}\right)+\chi(1 \in \mathcal{R})\right)}{1-z(x t) f\left(x, x t, \mathcal{D}^{+}, \mathcal{D}^{-}\right)}
\end{aligned}
$$


where

$$
z(t)= \begin{cases}t & \text { if } 0 \notin \mathcal{D} \\ \frac{t}{1-t} & \text { if } 0 \in \mathcal{D} .\end{cases}
$$

Remark 5 It may appear natural to attempt to solve (3.2), obtaining explicit functions as Carlitz did in (1.1). However, this presents two problems. Most obvious is the fact that we cannot do it except in the simplest cases. Less obvious is that doing so can make it more difficult to prove uniqueness of the singularity on the circle of convergence: For fixed $t>0$ the power series in the denominator in (3.2) has no positive coefficients except the constant term, whereas the behavior of the denominator of $f$ in (1.1) is unclear. (Compare the arguments for Carlitz compositions given in [10] and [11] with our use of Theorem 3 for the general case in the Sections 4 and 5.)

Proof: (Theorem 4) Let $\vec{c}$ be an $(\mathcal{L}, \mathcal{D}, \mathcal{R})$ composition. (Recall that, by definition, $\vec{c}$ has at least one part and all its parts are strictly positive.) Subtract 1 from every part. The remaining string of nonnegative integers has one or more alternating nonempty regions of zeros and nonzeros. We claim that this 1-reduction of $\vec{c}$ can be uniquely parsed as one of the two regular expressions

$$
X_{1} \quad \text { or } \quad\left(X_{2}+\epsilon_{\mathcal{L}}\right) Z\left(X_{3} Z\right)^{*}\left(X_{4}+\epsilon_{\mathcal{R}}\right) \text {. }
$$

where $Z$ denotes a nonempty string of zeros, $X_{i}$ a nonempty string of nonzeros, and

$$
\epsilon_{\mathcal{S}}= \begin{cases}\text { the empty string } \lambda, & \text { if } 1 \in \mathcal{S} \\ \text { nothing, } & \text { otherwise }\end{cases}
$$

(Thus, when $1 \notin \mathcal{S}, X+\epsilon_{\mathcal{S}}=X$ and, when $1 \in \mathcal{S}, X+\epsilon_{\mathcal{S}}=X \cup\{\lambda\}$.) To see this, note that $X_{1}$ covers the case "there are no zeros," while the second form is the case "there is at least one zero." Furthermore, we could have $c_{1}-1=0$ if and only if $1 \in \mathcal{L}$, which explains the presence of $\epsilon_{\mathcal{L}}$.

Not only does the 1-reduction of every $(\mathcal{L}, \mathcal{D}, \mathcal{R})$ composition have a unique parsing as described above, but there is also a converse: We will show how to uniquely construct all the $(\mathcal{L}, \mathcal{D}, \mathcal{R})$ compositions from such regular expressions. Start with any pattern of $X$ 's and $Z$ 's as in (3.4) and proceed as follows.

- If $0 \notin \mathcal{D}$, replace each $Z$ by one zero. If $0 \in \mathcal{D}$ replace each $Z$ by one or more zeros.

- Replace $X_{1}$ with any $\left(\mathcal{L}^{\prime}, \mathcal{D}, \mathcal{R}^{\prime}\right)$ composition.

- Replace $X_{2}$ with any $\left(\mathcal{L}^{\prime}, \mathcal{D}, \mathcal{D}^{-}\right)$composition.

- Replace each $X_{3}$ with any $\left(\mathcal{D}^{+}, \mathcal{D}, \mathcal{D}^{-}\right)$composition.

- Replace $X_{4}$ with any $\left(\mathcal{D}^{+}, \mathcal{D}, \mathcal{R}^{\prime}\right)$ composition.

- Finally, add 1 to every part. 
It is easily verified that the result is an $(\mathcal{L}, \mathcal{D}, \mathcal{R})$ composition and that every $(\mathcal{L}, \mathcal{D}, \mathcal{R})$ composition arises uniquely by such a process.

Now pass from the strings to their generating functions, noting the following.

- If $g(x, t)$ is a two-variable generating function for some set of compositions, then $g(x, x t)$ is the generating function for the set of compositions obtained when 1 is added to every part.

- $Z$ has generating function $z(t)$ given in the theorem.

- $\left(X_{3} Z\right)^{*}$ has generating function

$$
\frac{1}{1-z(t) f\left(x, t, \mathcal{D}^{+}, \mathcal{D}^{-}\right)} .
$$

This completes the proof.

\subsection{Rises and falls}

We can easily modify (3.2) to include variables to keep track of equality $\left(a_{i}=a_{i+1}\right)$, rises $\left(a_{i}<a_{i+1}\right)$ and falls. Introduce the vector $\left(t_{e}, t_{u}, t_{d}\right)$ for equality, rises and falls. If we count a rise at the first part and a fall at the last, then the rises plus falls plus equalities is one more than the number of parts. Thus at most two of the three variables $t_{e}, t_{d}, t_{u}$ should be introduced. Furthermore, if $0 \notin \mathcal{D}$, there are no equalities and so only one variable, either $t_{d}$ or $t_{u}$, should be introduced.

The adjustments to (3.2) are as follows. Set

$$
z(t)= \begin{cases}t & \text { if } 0 \notin \mathcal{D} \\ \frac{t}{1-t_{e} t} & \text { if } 0 \in \mathcal{D} .\end{cases}
$$

Replace $\chi(1 \in \mathcal{L})$ with $\chi(1 \in \mathcal{L}) t_{u}$ and $\chi(1 \in \mathcal{R})$ with $\chi(1 \in \mathcal{R}) t_{d}$.

\subsection{Local maxima and minima}

Given a composition, we add a part of 0 to each end for the purpose of counting local maxima. If $a_{i}<a_{i+1}=\cdots=a_{i+k}>a_{i+k+1}$, either

(a) we count this as one local maximum or

(b) we have $0 \in \mathcal{D}$ and we count this as $k$ local maxima.

(If $0 \notin \mathcal{D}$, we could never have $k>1$.) Likewise for local minima. In (a), local maxima and minima alternate, starting and ending with a local maximum. Thus there is exactly one more local maximum than local minima and so only one of the two counts should be made for a central or local limit theorem. In (b), there is no such dependence and so 
both counts can be made; however, $\max -1-\min$ is congruent to the number of equalities modulo 2 so the local limit theorem fails if maxima, minima and equalities are all counted.

Let $t_{M}$ count local maxima and $t_{m}$ count local minima. Define

$$
z(u, v)= \begin{cases}v z(u) & \text { in case (a) } \\ z(u v) & \text { in case (b) }\end{cases}
$$

where $z(t)$ is given by (3.3) or its modification in Section 3.2.

In the recursive construction, local maxima are introduced only in the composition $1, \ldots, 1$. Thus we subtract $\chi(1 \in \mathcal{L} \cap \mathcal{R}) z(x t)$ from the right side of (3.2) and add $\chi(1 \in \mathcal{L} \cap \mathcal{R}) z\left(x t, t_{M}\right)$.

In the recursive construction, local minima are introduced by the $1, \ldots, 1$ strings that appear between other parts. Thus the regular expression $\left(X_{2}+\epsilon_{\mathcal{L}}\right) Z\left(X_{3} Z\right)^{*}\left(X_{4}+\epsilon_{\mathcal{R}}\right)$ is broken into a sum of up to four terms:

$$
\begin{aligned}
\text { in all cases } & X_{2} Z_{1}\left(X_{3} Z_{1}\right)^{*} X_{4}, \\
\text { if } 1 \in \mathcal{L} & Z_{2}\left(X_{3} Z_{1}\right)^{*} X_{4}, \\
\text { if } 1 \in \mathcal{R} & X_{2}\left(Z_{1} X_{3}\right)^{*} Z_{2}, \\
\text { if } 1 \in \mathcal{L} \cap \mathcal{R} & Z_{2}\left(X_{3} Z_{1}\right)^{*} X_{3} Z_{2}+Z_{2}
\end{aligned}
$$

Then $Z_{2}$ is replaced by $z(x t)$ given by (3.3) and $Z_{1}$ is replaced by $z\left(x t, t_{m}\right)$. When the result is summed because of the ${ }^{*}$, we obtain the same fraction on the right of (3.2) with $z\left(x t, t_{m}\right)$ in the denominator, some minor corrections to the numerator plus correction terms similar to those for local maxima.

\subsection{Adjacent differences}

We can keep track of the number of times particular differences occur between adjacent parts. It may be easiest to assume that a composition begins and ends with zero. We keep track of differences other than zero, which is covered by $t_{e}$ in Section 3.2. If we are interested in a certain set $\mathcal{S}$ of differences, those elements of $\mathcal{L}, \mathcal{D}$ and $\mathcal{R}$ should be marked, say with $m_{s}$ for $s \in \mathcal{S}$. Two sets with the same elements are considered different if their marks differ. When the recursion (3.2) is applied, those marks are carried along. When a set $\mathcal{S}^{\prime}$ is constructed from $\mathcal{S}$, a mark on $s \in \mathcal{S}$ becomes a mark on $s-1 \in \mathcal{S}^{\prime}$. If $1 \in \mathcal{L}$ and 1 is marked $m_{s}$, then $\chi(1 \in \mathcal{L})$ is multiplied by $u_{s}$, a variable keeping track of an upward difference of $s$. Similarly with $\mathcal{R}$, using variables to keep track of the downward differences.

\subsection{Part sizes}

We introduce a variable $s_{i}$ to keep track of the number of parts of size $i$. If the largest part of interest has size $k$, we need $s_{1}, \ldots, s_{k}$ and set $s_{i}=1$ for $i>k$. Equation (3.2) must be modified as follows.

- Replace $\chi$ by $s_{0} \chi$ and $z(\alpha)$ with $z\left(\alpha s_{0}\right)$. 
- Apply $\mathfrak{S}$ to the right side of $(3.2)$, where $\mathfrak{S}$ replaces $s_{i}$ by $s_{i+1}$ for all $i$.

Since the recursion is obtained by (a) increasing each part size by 1 and (b) introducing new parts of size 1, it is easily seen that the generating function satisfies this recursion. Furthermore, if we start with the generating function $f$ that does not keep track of part size and iterate the recursion $k$ times, then we will be keeping track of all part sizes up to and including $k$.

Obviously we can set various $s_{i}$ equal after the above iteration; for example, $s_{i}=s$ for $i \in \mathcal{S}$ and $s_{i}=1$ otherwise. Thus $s$ keeps track of the number of parts in $\mathcal{S}$. This only works for finite $\mathcal{S}$. In the case of an infinite $\mathcal{S}$, such as counting the number of parts which are prime, one still gets normality. See [2].

\section{Basic Lemmas}

Definition 3 (some notation) Let $t_{1}$ keep track of the number of parts and $t_{2}, \ldots, t_{\ell}$ keep track of other things as discussed in Section 3. Think of $\mathbf{t}$ as a real valued vector near 1. In writing the generating functions, we may suppress $t_{2}, \ldots, t_{\ell}$ and replace $t_{1}$ with $t$.

Let $\operatorname{rad} g(x, \mathbf{t})$ be the radius of convergence, with respect to $x$, of the power series for $g(x, \mathbf{t})$.

If $\vec{c}$ is a composition, we denote the sum of its parts by $\Sigma(\vec{c})$.

To prove Theorem $1(\mathrm{a}-\mathrm{c})$ we want to show that Theorem 3 can be applied to the recursions (3.2) and the extensions discussed in Section 3. The application involves showing that, as a function of $x, f\left(x, x t_{1}, t_{2}, \ldots t_{\ell}\right)$ has a larger radius of convergence than $f(x, \mathbf{t})$ and then showing that (3.2) satisfies the hypotheses of Theorem 3. In this section we collect the necessary lemmas.

Lemma 2 Suppose $(\mathcal{L}, \mathcal{D}, \mathcal{R})$ is nontrivial. There exist $\lambda \in(0,1)$ and $\epsilon>0$, both depending only on $\mathcal{D}$, such that

$$
c_{\mathcal{L}, \mathcal{D}, \mathcal{R}}\left(n, k_{n}\right) \geq(1+\epsilon)^{n}
$$

for infinitely many pairs $\left(n, k_{n}\right)$ satisfying $k_{n} \geq \lambda n$.

Proof: Let $d^{+} \in \mathcal{D}^{+}, d^{-} \in \mathcal{D}^{-}$, and define $d=d^{+} d^{-}$. We will show by a construction that for each pair $\mathcal{L}, \mathcal{R}$ such that $(\mathcal{L}, \mathcal{D}, \mathcal{R})$ is nontrivial there exist constants $k_{0}, n_{0}, C_{0}$ such that for all sufficiently large $n$,

$$
c_{\mathcal{L}, \mathcal{D}, \mathcal{R}}\left(n C_{0}+n_{0}, 3 n\left(d^{-}+d^{+}\right)+k_{0}\right) \geq 2^{n},
$$

where $C_{0} \leq C_{1}$, a constant depending only on $\mathcal{D}$. Thus we may choose $\lambda=3\left(d^{-}+d^{+}\right) / C_{1}$. With $\epsilon$ defined by $1+\epsilon=2^{1 /\left(C_{1}+1\right)}$, we have

$$
2^{n}=\left(2^{n /\left(n C_{0}+n_{0}\right)}\right)^{n C_{0}+n_{0}} \geq\left(2^{n /\left(n C_{1}+n_{0}\right)}\right)^{n C_{0}+n_{0}} \geq\left(2^{1 /\left(C_{1}+1\right)}\right)^{n C_{0}+n_{0}}=(1+\epsilon)^{n C_{0}+n_{0}},
$$


the second inequality being true for all $n \geq n_{0}$. We now turn to the details of the construction. Let $a_{1}, \ldots, a_{k_{0}}$ be an $(\mathcal{L}, \mathcal{D}, \mathcal{R})$ composition with $k_{0}>1$ and call its sum $n_{0}$. We may assume that $a_{\ell} \leq d$ for some $\ell<k_{0}$ for, if not, consider

$$
a_{1}, b_{1}, \ldots, b_{m d^{+}}, c_{1}, \ldots, c_{m d^{-}}, a_{2}, \ldots, a_{k_{0}}
$$

where $m=\left\lfloor\left(a_{1}-1\right) / d\right\rfloor, b_{0}=a_{1}, b_{i+1}=b_{i}-d^{-}, c_{0}=b_{m d^{+}}$and $c_{i+1}=c_{i}+d^{+}$. Note that

$$
c_{m d^{-}}=c_{0}-\left(m d^{-}\right) d^{+}=b_{m d^{+}}-\left(m d^{-}\right) d^{+}=b_{0}+\left(m d^{+}\right) d^{-}-\left(m d^{-}\right) d^{+}=a_{1} .
$$

Define the up and down sequences

$$
\begin{aligned}
& \vec{v}(x):=x+d^{+}, x+2 d^{+}, \ldots, x+d^{-} d^{+} \\
& \vec{\delta}(x):=x-d^{-}, x-2 d^{-}, \ldots, x-d^{+} d^{-} .
\end{aligned}
$$

Consider the two sequences

$$
\begin{aligned}
& \vec{s}_{1}=\vec{v}\left(a_{\ell}\right), \vec{\delta}\left(a_{\ell}+d\right), \quad \vec{v}\left(a_{\ell}\right), \quad \vec{v}\left(a_{\ell}+d\right), \quad \vec{\delta}\left(a_{\ell}+2 d\right), \quad \vec{\delta}\left(a_{\ell}+d\right) \\
& \vec{s}_{2}=\vec{v}\left(a_{\ell}\right), \vec{v}\left(a_{\ell}+d\right), \vec{\delta}\left(a_{\ell}+2 d\right), \quad \vec{\delta}\left(a_{\ell}+d\right), \quad \vec{v}\left(a_{\ell}\right), \quad \vec{\delta}\left(a_{\ell}+d\right) .
\end{aligned}
$$

With $\Sigma(\vec{s})$ denoting the sum of the parts in $\vec{s}$,

$$
\begin{aligned}
\Sigma(\vec{v}(x)) & =d^{-} x+\frac{d\left(d^{-}+1\right)}{2} \\
\Sigma(\vec{\delta}(x)) & =d^{+} x-\frac{d\left(d^{+}+1\right)}{2} \\
\Sigma\left(\vec{s}_{1}\right)=\Sigma\left(\vec{s}_{2}\right) & =3\left(d^{+}+d^{-}\right) a_{\ell}+d^{-} d+4 d^{+} d+\frac{3 d\left(d^{-}-d^{+}\right)}{2} .
\end{aligned}
$$

The regular expression

$$
a_{1} \cdots a_{\ell}\left\{\vec{s}_{1}, \vec{s}_{2}\right\}^{n} a_{\ell+1} \cdots a_{k_{0}}
$$

gives $2^{n}$ different compositions of $n \Sigma\left(\vec{s}_{1}\right)+n_{0}$, each having $3 n\left(d^{-}+d^{+}\right)+k_{0}$ parts. Since $a_{\ell} \leq d, C_{0}=\Sigma\left(\vec{s}_{1}\right)$ is bounded by a function of $\mathcal{D}$, proving the lemma.

Lemma 3 Suppose $(\mathcal{L}, \mathcal{D}, \mathcal{R})$ is nontrivial and $t_{2}=\cdots=t_{\ell}=1$. For any $T>0$ and $R \in(0,1)$ there is a positive $\delta=\delta(T, R)$ independent of $(\mathcal{L}, \mathcal{D}, \mathcal{R})$, such that, if $0<t \leq T$ and $\operatorname{rad} f(x, t) \leq R$, then

$$
\operatorname{rad} f(x, x t) \geq(\operatorname{rad} f(x, t))(1+\delta) .
$$

Proof: Let $T>0$ and $R \in(0,1)$ be given, and let $\delta>0$ satisfy the two conditions

$$
(1+\delta) R^{1 /(1+\delta)}<1 \quad \text { and } \quad\left(\frac{e T}{\delta}\right)^{\delta} R^{1 /(1+\delta)}<1
$$

(The latter is possible since $\delta^{\delta} \rightarrow 1$ as $\delta \rightarrow 0^{+}$.) Let $(\mathcal{L}, \mathcal{D}, \mathcal{R})$ be a non trivial triple, $0<t \leq T$, and suppose that $\operatorname{rad} f(x, t)=r \leq R$. We will prove that the sum

$$
\sum_{n, k} c(n, k) x^{n+k} t^{k}
$$


converges for $0<x<r^{1 /(1+\delta)}$. It follows that $\operatorname{rad} f(x, x t) \geq r^{1 /(1+\delta)}$, and this is sufficient to establish the lemma because

$$
\min _{0<r \leq R} \frac{r^{1 /(1+\delta)}}{r}=\frac{R^{1 /(1+\delta)}}{R}=1+\hat{\delta}, \quad(\hat{\delta}>0) .
$$

To prove the asserted convergence, let $0<x<r^{1 /(1+\delta)}$, and split the sum into two parts. Using $c(n, k) \leq\left(\begin{array}{l}n-1 \\ k-1\end{array}\right)$, the number of unrestricted compositions of $n$ into $k$ parts,

$$
\begin{aligned}
\sum_{n, k} c(n, k) x^{n+k} t^{k} & =\sum_{n} x^{n} \sum_{k<\delta n} c(n, k)(x t)^{k}+\sum_{n} x^{n} \sum_{k \geq \delta n} c(n, k)(x t)^{k} \\
& \leq \sum_{n} x^{n} \sum_{k<\delta n}\left(\begin{array}{l}
n-1 \\
k-1
\end{array}\right)(x t)^{k}+\sum_{n, k} c(n, k) x^{(1+\delta) n} t^{k} \\
& =S_{1}+S_{2},
\end{aligned}
$$

say. Since $x^{1+\delta}<r$, we see immediately that $S_{2}<\infty$. As for $S_{1}$, if $x t \leq \delta$ then $S_{1} \leq \delta \sum_{n}(1+\delta)^{n-1} x^{n}<\infty$ by the first condition in (4.3). Otherwise $x t>\delta$, and

$$
\left(\begin{array}{l}
n-1 \\
k-1
\end{array}\right)(x t)^{k}=\frac{k}{n}\left(\begin{array}{l}
n \\
k
\end{array}\right)(x t)^{k} \leq \frac{k}{n} \frac{(n x t)^{k}}{k !} \leq \frac{k}{n}\left(\frac{e n x t}{k}\right)^{k},
$$

the last by $k ! \geq(k / e)^{k}$. The function $k \mapsto(e n x t / k)^{k}$ is an increasing function of a real variable $k$ in the range $1 \leq k<n x t$. Since $x t>\delta$,

$$
\sum_{1 \leq k<\delta n}\left(\begin{array}{l}
n-1 \\
k-1
\end{array}\right)(x t)^{k}<\delta n \frac{\delta n}{n}\left(\frac{e n x t}{\delta n}\right)^{\delta n}=\delta^{2} n\left(\frac{e x t}{\delta}\right)^{\delta n},
$$

and again $S_{1}$ is finite, this time by the second condition in (4.3).

Lemma 4 Suppose $\operatorname{gcd}(\mathcal{D})=1$ and $t_{2}=\cdots=t_{\ell}=1$. Define $r(t)$ by $r(t)=\inf _{\mathcal{L}, \mathcal{R}}\{\operatorname{rad} f(x, t, \mathcal{L}, \mathcal{D}, \mathcal{R})\}$. Then, whenever $t \geq 1$,

(a) $0<r(t)<1$,

(b) $\operatorname{rad} f(x, t, \mathcal{L}, \mathcal{D}, \mathcal{R})=r(t)$ for all $\mathcal{L}$ and $\mathcal{R}$,

(c) $z(r(t)) f\left(r(t), r(t), \mathcal{D}^{+}, \mathcal{D}^{-}\right)=1$,

(d) $\operatorname{rad} f(x, x t, \mathcal{L}, \mathcal{D}, \mathcal{R})>r(t)$.

Proof: Since there are at most $2^{n-1}$ compositions of $n, r(t) \geq 1 / 2 t$. By Lemma 1 every triple $(\mathcal{L}, \mathcal{D}, \mathcal{R})$ is nontrivial. By Lemma 2 there is an $\epsilon>0$, depending only on $\mathcal{D}$, such that $\operatorname{rad} f(x, 1, \mathcal{L}, \mathcal{D}, \mathcal{R}) \leq(1+\epsilon)^{-1}$. Since $r(t)$ is clearly a nondecreasing function of $t$, this proves $(\mathrm{a})$.

Choose $R \in\left(\frac{1}{1+\epsilon}, 1\right)$ and let $\delta=\delta(t, R)$ be given by Lemma 3. Thus

$$
\operatorname{rad} f(x, x t, \mathcal{L}, \mathcal{D}, \mathcal{R}) \geq \operatorname{rad} f(x, t, \mathcal{L}, \mathcal{D}, \mathcal{R})(1+\delta) \geq r(t)(1+\delta) .
$$


Choose $\mathcal{L}_{0}, \mathcal{R}_{0}$ so that

$$
\operatorname{rad} f\left(x, t, \mathcal{L}_{0}, \mathcal{D}, \mathcal{R}_{0}\right)<(1+\delta) r(t) .
$$

Call this radius of convergence $r_{0}(t)$. Since $f\left(x, t, \mathcal{L}_{0}, \mathcal{D}, \mathcal{R}_{0}\right)$ has nonnegative coefficients, it has a singularity at $x=r_{0}(t)$. Consider equation $(3.2)$ with $f\left(x, t, \mathcal{L}_{0}, \mathcal{D}, \mathcal{R}_{0}\right)$ on the left side. All $f$ 's on the right side have radii of convergence greater than or equal to $(1+\delta) r(t)$ by (4.4). The only way this can happen - for the power series on the right to have radii greater than $r_{0}(t)$ and $f\left(x, t, \mathcal{L}_{0}, \mathcal{R}_{0}\right)$ to have a singularity at $r_{0}(t)$ - is for the denominator on the right, $1-z(x t) f\left(x, x t, \mathcal{D}^{+}, \mathcal{D}, \mathcal{D}^{-}\right)$, to vanish at $x=r_{0}(t)$. Since the coefficients of $z(x t) f\left(x, x t, \mathcal{D}^{+}, \mathcal{D}, \mathcal{D}^{-}\right)$are nonnegative, this is the unique positive zero of the denominator. The coefficients of the factors in the numerator are nonnegative, and so there can be no cancellation due to vanishing of the numerator. Thus $r_{0}(t)$ is independent of $\mathcal{L}_{0}$ and $\mathcal{R}_{0}$. Taking the infimum over all $\mathcal{L}_{0}, \mathcal{R}_{0}$ satisfying (4.5), $r(t)=r_{0}(t)$ and so (c) holds. It follows that every generating function $f(x, t, \mathcal{L}, \mathcal{D}, \mathcal{R})$ has the same radius of convergence, $r(t)$, proving (b). By Lemma $3, \operatorname{rad} f(x, x t, \mathcal{L}, \mathcal{R})>\operatorname{rad} f(x, t, \mathcal{L}, \mathcal{R})$, which equals $r(t)$, proving (d).

Lemma 5 Suppose $\operatorname{gcd}(\mathcal{D})=1$. There exist constants $\rho>r>0$ and $\tau>1$ such that for each pair $\mathcal{L}, \mathcal{R}$ there are power series $F(x, \mathbf{t}), G(x, \mathbf{t})$, and $H(x, \mathbf{t})$ satisfying

(a) $f(x, \mathbf{t}, \mathcal{L}, \mathcal{D}, \mathcal{R})=F(x, \mathbf{t})+\frac{G(x, \mathbf{t})}{1-H(x, \mathbf{t})}$,

(b) $H(r, \mathbf{1})=1$,

(c) $G(r, \mathbf{1}) \neq 0$,

(d) the series for $F, G$ and $H$ converge whenever $|x|<\rho$ and $\left|t_{i}\right|<\tau$.

Proof: Let $F, G, H$ be the three power series on the right side of equation (3.2): the summand, the numerator, and 1 minus the denominator, respectively. Let $r(t)$ be as in Lemma 4 and let $r=r(1)$. By that lemma, $\operatorname{rad} G(x, \mathbf{1})>r, \operatorname{rad} H(x, \mathbf{1})>r$ and $H(r, \mathbf{1})=1$. Since its coefficients are nonnegative, $G(r, 1) \neq 0$.

It remains to prove (d) by finding appropriate $\rho$ and $\tau$. Let $\mathbf{t}_{x}=\left(x t_{1}, t_{2}, \ldots, t_{\ell}\right)$. By the nature of what the $t_{i}$ count, no $t_{i}$ appears to a higher power than $n$ in $\left[x^{n}\right] f\left(x, \mathbf{t}_{x}\right)$. Hence $\left|\left[x^{n}\right] f\left(x, \mathbf{t}_{x}\right)\right| \leq\left|\left[x^{n}\right] f\left(x, \mathbf{1}_{x}\right)\right| \tau^{n \ell}$. Thus, with $\delta=\delta(1, r)$ given by Lemma 3 ,

$$
\operatorname{rad} f\left(x, \mathbf{t}_{x}\right) \geq \tau^{-\ell} \operatorname{rad} f\left(x, \mathbf{1}_{x}\right) \geq \tau^{-\ell}(1+\delta) \operatorname{rad} f(x, \mathbf{1})=\tau^{-\ell}(1+\delta) r .
$$

Let $\tau=(1+\delta)^{1 / 2 \ell}$ and $\rho=r(1+\delta)^{1 / 2}$.

\section{Proofs of Theorem $1(\mathrm{a}-\mathrm{c})$ and Theorem 2}

To establish Theorem $1(\mathrm{a}-\mathrm{c})$, we will show that Theorem 3 can be applied to the right side of (3.2) with $H(x, \mathbf{t})=z f\left(x, \mathbf{t}_{x}, \mathcal{D}^{+}, \mathcal{D}^{-}\right)$, where $\mathbf{t}_{x}=\left(x t_{1}, t_{2}, \ldots, t_{\ell}\right)$ and $z$ is either $z(x t)$ or an appropriate modification of it as in Section 3.2, 3.3 or 3.5. 
Note that either of the conditions on $\mathcal{D}$ stated in the first sentence of Theorem 1 implies that $\operatorname{gcd}(\mathcal{D})=1$. The existence of $\rho, r, \tau$ and conditions (a)-(c) of Theorem 3 are thus established by Lemma 5. Part sizes require a bit of care since, as discussed in Section 3.5, the variables counting parts of a given size change from one side of (3.2) to the other. This is not a problem because the only time these variables are not equal to 1 is in the latter half of the proof of Lemma 5 .

Condition (d) of Theorem 3 holds because, by definition, the nonzero terms $c(n, k) x^{n} t^{k}$ of our generating functions occur only for $n>0$.

To prove Theorem 1(a,b), we can assume that $\ell=1$; that is, only the number of parts in a composition of $n$ is being counted.

We first show that $\operatorname{gcd}\left(\mathcal{D}^{+} \cap \mathcal{D}^{-}\right)=1$ implies Theorem $3(\mathrm{e}, \mathrm{f})$. Let $d \in \mathcal{D}^{+} \cap \mathcal{D}^{-}$ be odd. Consider the compositions $d$ and $d, 2 d, d$. Since $z(t)$ contains the term $t$, $z(x t) f(x, x t, \mathcal{D}, \mathcal{D})$ contains the terms

$$
(x t) x^{d}(x t)=x^{d+2} t^{2} \text { and }(x t) x^{4 d}(x t)^{3}=x^{4 d+4} t^{4} .
$$

Thus

$$
(d+2,2) \in \mathcal{H}_{d+2} \quad \text { and } \quad(4 d+4,4) \in \mathcal{H}_{4 d+4} .
$$

Using the fact that $d$ is odd, condition (e) follows since

$$
\begin{aligned}
\operatorname{gcd}\left\{n \mid \mathcal{H}_{n} \neq \emptyset\right\} & \leq \operatorname{gcd}(d+2,4 d+4)=\operatorname{gcd}(d+2,4(d+2)-(4 d+4)) \\
& =\operatorname{gcd}(d+2,4)=1,
\end{aligned}
$$

Condition (f) follows from (5.1) since det $\left|\begin{array}{cc}d+2 & 2 \\ 4 d+4 & 4\end{array}\right|=-4 d \neq 0$.

We now verify that Theorem $3(\mathrm{e}-\mathrm{g})$ hold when $\operatorname{gcd}(\mathcal{D}-\mathcal{D})=1$. Let $\mathcal{S}$ be the span of $\mathcal{H}$ over $\mathbb{Z}$. We first show that $(1,0) \in \mathcal{S}$, by two cases depending on whether $0 \in \mathcal{D}$ or not. This implies (e) and (f), the latter since $(n, k) \in \mathcal{H}$ for some $n$ and $k \neq 0$.

Suppose $0 \in \mathcal{D}$. For $d^{+} \in \mathcal{D}^{+}$and $d^{-} \in \mathcal{D}^{-}$, consider the two compositions

$$
\begin{aligned}
& d^{+}, \quad d^{+}, \quad 2 d^{+}, \ldots,\left(2 d^{-}-1\right) d^{+}, 2 d^{-} d^{+},\left(2 d^{+}-1\right) d^{-},\left(2 d^{+}-2\right) d^{-}, \ldots, d^{-} \\
& d^{+}, 2 d^{+}, 2 d^{+}, \ldots,\left(2 d^{-}-1\right) d^{+}, 2 d^{-} d^{+},\left(2 d^{+}-1\right) d^{-},\left(2 d^{+}-2\right) d^{-}, \ldots, d^{-}
\end{aligned}
$$

Since they have the same number of parts $k$ and differ in sum by $d^{+}$, it follows that $\left(n+d^{+}, k\right)-(n, k)=\left(d^{+}, 0\right) \in \mathcal{S}$. Similarly $\left(d^{-}, 0\right) \in \mathcal{S}$ and so $(1,0) \in \mathcal{S}$ since $\operatorname{gcd}(\mathcal{D})=1$ by Remark 3 .

If $0 \notin \mathcal{D}$, choose $d^{+} \in \mathcal{D}^{+}$and $d^{-} \in \mathcal{D}^{-}$and consider the two compositions

$$
\begin{aligned}
& d^{+}, 2 d^{+}, \ldots,\left(2 d^{-}-1\right) d^{+}, \quad 2 d^{-} d^{+}, \quad\left(2 d^{+}-1\right) d^{-},\left(2 d^{+}-2\right) d^{-}, \ldots, d^{-} \\
& d^{+}, 2 d^{+}, \ldots,\left(2 d^{-}-1\right) d^{+},\left(2 d^{-}-1\right) d^{+}-d^{-},\left(2 d^{+}-1\right) d^{-},\left(2 d^{+}-2\right) d^{-}, \ldots, d^{-} \text {. }
\end{aligned}
$$

Since they have the same number of parts $k$ and differ in sum by $d^{+}+d^{-}$, it follows that $\left(n+d^{+}+d^{-}, k\right)-(n, k)=\left(d^{+}+d^{-}, 0\right) \in \mathcal{S}$. We now show this implies that $(1,0) \in \mathcal{S}$. 
For $d_{1}^{+}, d_{2}^{+} \in \mathcal{D}^{+}$we can write $d_{1}^{+}-d_{2}^{+}$as the difference of two elements in $\mathcal{D}^{+}+\mathcal{D}^{-}$, namely $d_{1}^{+}-d_{2}^{+}=\left(d_{1}^{+}+d^{-}\right)-\left(d_{2}^{+}+d^{-}\right)$. Likewise for two elements of $\mathcal{D}^{-}$. Since

$$
\mathcal{D}-\mathcal{D}= \pm\left(\mathcal{D}^{+}+\mathcal{D}^{-}\right) \cup\left(\mathcal{D}^{+}-\mathcal{D}^{+}\right) \cup\left(\mathcal{D}^{-}-\mathcal{D}^{-}\right),
$$

it follows that $\operatorname{gcd}(\mathcal{D}-\mathcal{D})=\operatorname{gcd}\left(\mathcal{D}^{+}+\mathcal{D}^{-}\right)$. By assumption, $\operatorname{gcd}(\mathcal{D}-\mathcal{D})=1$. Thus $(1,0) \in \mathcal{S}$.

We now verify $\mathcal{S}=\mathbb{Z}^{2}$ to establish the local limit part of Theorem 1(b). Since $(1,0) \in \mathcal{S}$, it suffices to show that

$$
\operatorname{gcd}\left\{k+1 \mid c_{\mathcal{D}^{+}, \mathcal{D}^{-}}(n, k) \neq 0\right\}=1 .
$$

If $0 \in \mathcal{D}$, this is trivial since we can always repeat a part in a composition with $k$ parts to get a composition with $k+1$ parts. If $0 \notin \mathcal{D}$, the composition

$$
d^{+}, 2 d^{+}, \ldots,\left(d^{-}-1\right) d^{+}, d^{-} d^{+},\left(d^{+}-1\right) d^{-}, \ldots, d^{-}
$$

has $k+1=d^{+}+d^{-}$and, by the previous paragraph, we know that $\operatorname{gcd}\left(\mathcal{D}^{+}+\mathcal{D}^{-}\right)=1$. This completes the proof of Theorem $1(\mathrm{a}, \mathrm{b})$. We have also verified Theorem 3(e) in general.

We now turn to Theorem 1(c). Since Theorem 3(a-e) have been verified, only (f) and (g) remain. This is a matter of exhibiting enough compositions to verify that $\mathcal{H}$ spans either $\mathbb{R}^{\ell+1}$ (for a central limit theorem) or $\mathbb{Z}^{\ell+1}$ (for a local limit theorem), remembering to include the number of parts as one of the variables. (This is what "nondegenerate" meant in the statement of the theorem.) This is usually straightforward for specific $\mathcal{D}$ and variables being counted; however, it may be difficult to establish a result for an entire class of cases. Particularly easy are the cases of all compositions and Carlitz compositions because of the great freedom provided by $\mathcal{D}$.

We conclude this section with a proof of Theorem 2. Apply Lemma 1. Since all elements of $\mathcal{D}$ are multiples of $\delta, f\left(x, t, \mathcal{L}_{i}, \mathcal{D}\right)=f\left(x, t, \mathcal{D}, \mathcal{R}_{i}\right)=0$ for $1 \leq i<\delta$. Hence (3.2) gives

$$
\begin{aligned}
f\left(x, t, \mathcal{L}_{i}, \mathcal{R}_{i}\right) & =f\left(x, x t, \mathcal{L}_{i-1}, \mathcal{R}_{i-1}\right) \quad \text { for } 1<i<\delta \\
f\left(x, t, \mathcal{L}_{1}, \mathcal{R}_{1}\right) & =\frac{z(x t)}{1-z(x t) f\left(x, x t, \mathcal{D}^{+}, \mathcal{D}^{-}\right)} .
\end{aligned}
$$

Thus we have

$$
f\left(x, t, \mathcal{L}_{i}, \mathcal{R}_{i}\right)=\frac{z\left(x^{i} t\right)}{1-z\left(x^{i} t\right) f\left(x, x^{i} t, \mathcal{D}^{+}, \mathcal{D}^{-}\right)} .
$$

One can apply Theorem 3 to this. The radii of convergence $r_{i}$ are the positive solutions of $1-z\left(r_{i}^{i}\right) f\left(r_{i}, r_{i}^{i}, \mathcal{D}^{+}, \mathcal{D}^{-}\right)=0$, and so $r_{i}>r_{i-1}$. The construction leading to (5.1) gives us

$$
(d+2 i, 2) \in \mathcal{H}_{d+2 i} \quad \text { and } \quad(4 d+4 i, 4) \in \mathcal{H}_{4 d+4 i} .
$$


Thus

$$
\begin{aligned}
\operatorname{gcd}\left\{n \mid \mathcal{H}_{n} \neq \emptyset\right\} & \leq \operatorname{gcd}\left\{d+2 i, 4 d+4 i \mid d \in \mathcal{D}^{-}\right\} \\
& =\operatorname{gcd}\left\{d+2 i, 4(d+2 i)-(4 d+4 i),(4 d+4 i)-2(d+2 i) \mid d \in \mathcal{D}^{-}\right\} \\
& =\operatorname{gcd}\left\{d+2 i, 4 i, 2 d \mid d \in \mathcal{D}^{-}\right\}=\operatorname{gcd}\left\{d+2 i, 4 i, 2 \delta \mid d \in \mathcal{D}^{-}\right\} \\
& =\operatorname{gcd}\left\{d+2 i, 2 \mid d \in \mathcal{D}^{-}\right\}=\operatorname{gcd}\left\{d, 2 \mid d \in \mathcal{D}^{-}\right\} \\
& =\operatorname{gcd}\{\delta, 2\}=1,
\end{aligned}
$$

where we have used the facts that $1 \leq i<\delta=\operatorname{gcd}\left(\mathcal{D}^{-}\right)$and $\delta$ is an odd prime.

\section{Proof of Theorem 1(d)}

Add variables $u$ and $v$ to $f$ to keep track of first and last parts. When this is done, (3.2) becomes

$$
\begin{aligned}
& f(x, t, u, v, \mathcal{L}, \mathcal{R})=f\left(x, x t, u, v, \mathcal{L}^{\prime}, \mathcal{R}^{\prime}\right) u v \\
& +\frac{\left(f\left(x, x t, u, 1, \mathcal{L}^{\prime}, \mathcal{D}\right) u+\chi(1 \in \mathcal{L}) u\right) z(x t)\left(f\left(x, x t, 1, v, \mathcal{D}, \mathcal{R}^{\prime}\right) v+\chi(1 \in \mathcal{R}) v\right)}{1-z(x t) f\left(x, x t, \mathcal{D}^{+}, \mathcal{D}^{-}\right)}
\end{aligned}
$$

As usual, let $r$ be the radius of convergence of $f(x, 1, \mathcal{L}, \mathcal{R})$. We claim that the radius of convergence of $f(x, x, u, v, \mathcal{L}, \mathcal{R})$ exceeds $r$ provided $|u|$ and $|v|$ are not much larger that 1 , say less than $1+\epsilon$. This follows from two facts. First, no single part can exceed the sum of the parts. Second, by Lemma $3, f(x, x, \mathcal{L}, \mathcal{R})$ has radius of convergence larger than $r$.

The joint distribution for $u$ and $v$ is $J_{n}(u, v)=\frac{\left[x^{n}\right] f(x, 1, u, v, \mathcal{L}, \mathcal{R})}{\left[x^{n}\right] f(x, 1,1,1, \mathcal{L}, \mathcal{R})}$. Set $t=1$ in $(6.1)$ and assume $|u|,|v|<1+\epsilon$. By the previous paragraph, all occurrences of $f$ on the right side of (6.1) have radius of convergence exceeding $r$. Thus we are still dealing with a simple pole and so we obtain

$$
\begin{aligned}
J_{n}(u, v) & \sim \frac{\left(f\left(r, r, u, 1, \mathcal{L}^{\prime}, \mathcal{D}\right) u+\chi(1 \in \mathcal{L}) u\right)\left(f\left(r, r, 1, v, \mathcal{D}, \mathcal{R}^{\prime}\right) v+\chi(1 \in \mathcal{R}) v\right)}{\left(f\left(r, r, 1,1, \mathcal{L}^{\prime}, \mathcal{D}\right)+\chi(1 \in \mathcal{L})\right)\left(f\left(r, r, 1,1, \mathcal{D}, \mathcal{R}^{\prime}\right)+\chi(1 \in \mathcal{R})\right)} \\
& =\frac{f\left(r, r, u, 1, \mathcal{L}^{\prime}, \mathcal{D}\right) u+\chi(1 \in \mathcal{L}) u}{f\left(r, r, 1,1, \mathcal{L}^{\prime}, \mathcal{D}\right)+\chi(1 \in \mathcal{L})} \frac{f\left(r, r, 1, v, \mathcal{D}, \mathcal{R}^{\prime}\right) v+\chi(1 \in \mathcal{R}) v}{f\left(r, r, 1,1, \mathcal{D}, \mathcal{R}^{\prime}\right)+\chi(1 \in \mathcal{R})},
\end{aligned}
$$

a product of distributions. One could also compute partials with respect to $u$ and/or $v$ before carrying out the asymptotics, which would lead to the corresponding partials in (6.2). Thus all moments are finite since $f\left(r, r, u, 1, \mathcal{L}^{\prime}, \mathcal{D}\right)$ is analytic for $|u|<1+\epsilon$ and $f\left(r, r, 1, v, \mathcal{D}, \mathcal{R}^{\prime}\right)$ is analytic for $|v|<1+\epsilon$. 


\section{Upper Bounds on $D_{n}$ and $M_{n}$}

Since $D_{n} \leq M_{n}$, an upper bound on $M_{n}$ is an upper bound on $D_{n}$, it suffices to prove upper bounds on $M_{n}$.

We begin with the upper bound in Theorem $1(\mathrm{e})$. Suppose $a_{1}, \ldots, a_{k}$ is an $(\mathcal{L}, \mathcal{D}, \mathcal{R})$ composition of $n$ in which $a_{i}=\ell$ for some $1<i<k$. All such compositions can be built by listing an $(\mathcal{L}, \mathcal{D}, \mathbb{N})$ composition $a_{1}, \ldots, a_{i-1}$ for some $i$, the part $\ell$ and then an $(\mathbb{N}, \mathcal{D}, \mathcal{R})$ composition $a_{i+1}, \ldots, a_{k}$. Not all compositions built this way need be $(\mathcal{L}, \mathcal{D}, \mathcal{R})$ compositions and those with more than one large part will be counted more than once, but this is of no concern since we want an upper bound on the number. Letting $a_{1}+\cdots+a_{i-1}=t$, it follows from Theorem 1(a) that the number of such compositions is bounded above by $\left(C_{1} r^{-t}\right)\left(C_{1} r^{-(n-t-\ell)}\right)$ for some $C_{1}>0$. Since $k \leq n$, the number of $(\mathcal{L}, \mathcal{D}, \mathcal{R})$ compositions having at least one part of size at least $L$ is bounded above by

$$
\sum_{\ell \geq L} \sum_{t=0}^{n-\ell}\left(C_{1} r^{-t}\right)\left(C_{1} r^{-(n-t-\ell)}\right) \leq C_{1}^{2} n r^{-n} \sum_{\ell \geq L} r^{\ell} \leq C_{2} n r^{-n} r^{L}
$$

since $r<1$. To prove the upper bound, it suffices to have $L>\log _{1 / r}(n)+g(n)$ for any function $g(n) \rightarrow \infty$.

The upper bound in Theorem 1(f) requires more work and the remainder of this section is devoted to it. Our proof could be adapted to obtain results between $(\log n)^{1 / 2}$ and $\log n$ for some cases when $\mathcal{D}^{+}$and $\mathcal{D}^{-}$are infinite but much sparser than allowed in $(\mathrm{g})$.

We begin with a lemma whose proof is immediate from Theorem 1(a).

Lemma 6 Let $\mathcal{D}$ satisfy the hypotheses of Theorem 1. Define $\mathcal{S}_{t}=\{s \in \mathcal{S} \mid s<t\}$. Let $c_{t}(n)$ be the number of $\left(\mathcal{L}_{t}, \mathcal{D}, \mathcal{R}_{t}\right)$ compositions of $n$. Thus $c_{\infty}(n)$ is the number of $(\mathcal{L}, \mathcal{D}, \mathcal{R})$ compositions of $n$. It follows that $c_{t+1}(n) \geq c_{t}(n)$ and, in the notation of Theorem 1(a), $A\left(\mathcal{L}_{t}, \mathcal{D}, \mathcal{R}_{t}\right) \rightarrow A(\mathcal{L}, \mathcal{D}, \mathcal{R})$ as $t \rightarrow \infty$. Thus, if $t \rightarrow \infty$ with $n$, no matter how slowly, $c_{t}(n) / c_{\infty}(n) \rightarrow 1$.

By the lemma, it suffices to study the largest part in $\left(\mathcal{L}, \mathcal{D}, \mathcal{R}_{t}\right)$ compositions provided we let $t \rightarrow \infty$. By symmetry, we can assume that $\mathcal{D}^{-}$is finite. Let $d=\max \mathcal{D}^{-}$and let $h=C_{1}(\log n)^{1 / 2}$ where $C_{1}>0$ will be specified later. Let $t \rightarrow \infty$ but $t<h / 2-d$. We now bound the number of $\left(\mathcal{L}, \mathcal{D}, \mathcal{R}_{t}\right)$ compositions containing a part larger than $h$. They are contained in the multiset of compositions $\vec{a} \vec{b} \vec{c}$ where $b_{1}>h, c_{1} \leq h / 2$ and all $b_{i}$ exceed $h / 2$. The value of $t$ insures that $\vec{c}$ is not empty; however $\vec{a}$ may be empty. By the definition of $d$, it follows from $b_{1}-c_{1}>h / 2$ that $\vec{b}$ has at least $h / 2 d$ parts. Let $\vec{d}$ be $\vec{b}$ with $h / 2$ subtracted from each part and let $\vec{d}$ have $k$ parts. It follows that

$$
n=\Sigma(\vec{a} \vec{b} \vec{c})=\Sigma(\vec{a})+\Sigma(\vec{d})+\Sigma(\vec{c})+(h / 2) k \geq \Sigma(\vec{a})+\Sigma(\vec{d})+\Sigma(\vec{c})+(h / 2)(h / 2 d) .
$$

Since the number of $(\mathbb{N}, \mathcal{D}, \mathbb{N})$ compositions $\vec{f}$ is at most $C_{2} r^{-\Sigma(\vec{f})}$. the number of compositions obtained in this manner is at most

$$
\sum C_{2}^{3} r^{-\Sigma(\vec{a})+\Sigma(\vec{d})+\Sigma(\vec{c})} \leq \sum C_{2}^{3}-n+h^{2} / 4 d
$$


where the sum is over all ways to choose $\Sigma(\vec{a}), \Sigma(\vec{b})$ and $\Sigma(\vec{c})$. The number of choices is bounded by $n^{2}$. Thus we construct at most $C_{2}^{3} r^{-n}\left(n^{2} r^{h^{2} / 4 d}\right)$ compositions. Since there are at least $C_{3} r^{-n}(\mathcal{L}, \mathcal{D}, \mathcal{R})$ compositions for large $n$, we are done provided $n^{2} r^{h^{2} / 4 d} \rightarrow 0$, which will happen if $h \geq C_{1}(\log n)^{1 / 2}$ and $C_{1}$ is sufficiently large.

\section{Lower Bounds for $D_{n}$ and $M_{n}$}

In this discussion the $C_{i}$ are positive constants whose explicit values are not of concern.

We claim that there is a $\tau>0$ so that almost all $(\mathcal{L}, \mathcal{D}, \mathcal{R})$ compositions of $n$ have at least $\tau n$ parts of size 1. Given this, it suffices to prove lower bounds in Theorem 1(e,f) for such compositions. To prove the claim, we to apply Theorem 1(c), keeping track of parts of size 1. By Section 3.5, the relevant denominator is $1-z\left(x s_{1}\right) f(x, x)$. For every composition of $n$ with $k$ parts we obtain at least a term $x s_{1} x^{n} x^{k}=x^{n+k+1} s_{1}$. Since the vectors $(n+k+1,1)$ span $\mathbb{R}^{2}$, we are done.

Let $\mathcal{S}_{n}(p)$ be the number of $(\mathcal{L}, \mathcal{D}, \mathcal{R})$ compositions of $n$ having at least $\tau n$ parts of size 1 and no parts of size $p$. Suppose we have a $(\{1\}, \mathcal{D},\{1\})$ composition of $\sigma(p)+1$ containing a part of size $p$. Let $\vec{c}$ be this composition with the first part removed. If $a_{1}, \ldots, a_{k}$ is a composition of $n$ not containing $p$, we can insert $\vec{c}$ immediately after any $a_{i}=1$. This produces at least $\tau n\left|\mathcal{S}_{n}(p)\right|$ distinct $(\mathcal{L}, \mathcal{D}, \mathcal{R})$ compositions of $n+\sigma(p)$. Since there are less than $C_{1} r^{-n-\sigma(p)}$ such compositions for some $C_{1}>0$,

$$
\left|\mathcal{S}_{n}(p)\right|<C_{2} r^{-n}\left(\frac{1}{n r^{\sigma(p)}}\right) .
$$

If $p=p(n)$ is such that $n r^{\sigma(p)} \rightarrow \infty$, it will follow that a composition almost surely contains a part of size $p$. If

$$
\sum_{\mathcal{P}(n)} r^{-\sigma(p)}=o(n)
$$

it follows that the probability a composition does not contain any parts in $\mathcal{P}(n)$ is $o(1)$. Appropriate choices of $\mathcal{P}(n)$ will then complete the proofs of Theorem $1(\mathrm{f}, \mathrm{g})$.

We begin with (f). Choose any $d^{+} \in \mathcal{D}^{+}$and $d^{-} \in \mathcal{D}^{-}$and keep them fixed. Let $d=d^{+} d^{-}$. Recall the definitions in (4.1). Let $p=k d+1$ and

$$
1, \vec{c}=\vec{v}(1), \vec{v}(1+d), \ldots, \vec{v}(1+(k-1) d), \vec{\delta}(1+k d), \vec{\delta}(1+(k-1) d), \ldots, \vec{\delta}(1+d)
$$

According to (4.2), the sum of the parts is

$$
\begin{aligned}
\sigma(p) & =\sum_{i=0}^{k-1} \Sigma(\vec{v}(1+i d))+\sum_{i=1}^{k} \Sigma(\vec{\delta}(1+i d))-1 \\
& =\sum_{i=0}^{k-1}\left(d^{-}(1+i d)+\frac{d\left(d^{-}+1\right)}{2}\right)+\sum_{i=1}^{k}\left(d^{+}(1+i d)-\frac{d\left(d^{+}+1\right)}{2}\right)-1 \\
& \leq C_{3} k^{2}
\end{aligned}
$$


Let $\mathcal{P}(n)=\left\{k d+1 \mid 1<k<\left(C_{4} \log n\right)^{1 / 2}\right\}$. The left side of (8.2) is bounded above by

$$
\sum_{k} r^{-C_{3} k^{2}}<\left(C_{4} \log n\right)^{1 / 2} r^{-C_{3} C_{4} \log n}
$$

It suffices to choose $C_{4}$ so that $C_{3} C_{4} \log (1 / r)<1$. This proves that $D_{n} \geq C_{5}(\log n)^{1 / 2}$ with probability $1-o(1)$. This completes the proof of $(\mathrm{f})$.

We now turn our attention to the lower bounds in $(\mathrm{g})$. If $\rho>0$, we simply let $\vec{c}=(d+1), 1$, where $d \in \mathcal{D}^{+} \cap \mathcal{D}^{-}$. Equation (8.1) requires that $\log _{1 / r} n-d \rightarrow \infty$. Since $d \in \mathcal{D}^{+} \cap \mathcal{D}^{-}$, the largest possible choice for $d$ may differ from $\log _{1 / r} n$ by as much as a factor asymptotic to $\rho$. This proves the claim for $M_{n}$ in (g.ii) when $B=\rho$.

The value $B=\rho^{+} \rho^{-}$requires a more complicated construction. There is a constant $C$ such that, for all $k \leq \min \left(\mathcal{D}^{-}\right)$there is an $n=n(k) \leq C$ such that there is an $(k, \mathcal{D},\{1\})$ composition of $n$. Let $\vec{r}(k)$ be this composition with its first part $k$ removed. Similarly for $k \leq \min \left(\mathcal{D}^{+}\right)$and any $(\{1\}, \mathcal{D}, k)$ composition, let $\vec{\ell}(k)$ be this composition with its first part 1 and last part $k$ both removed. (Some compositions may be empty.)

For $p-1 \in \mathcal{D}^{+}$, we construct a composition $\vec{c}=c_{1}, c_{2}, \ldots, c_{t}, \vec{r}\left(c_{t}\right)$, where $c_{1}=p$, $c_{i}-c_{i-1}$ is as large as possible and $c_{t} \leq \min \left(\mathcal{D}^{-}\right)$. Suppose $c_{i+1}-c_{i}=d_{j}$ in the ordering of $\mathcal{D}^{-}$in Theorem 1(f). By assumption, $c_{i+1} \leq d_{j+1}$. Thus

$$
c_{i}=c_{i+1}-d_{j}=c_{i+1}\left(1-\frac{d_{j}}{c_{i+1}}\right) \leq c_{i+1}\left(1-\frac{d_{j}}{d_{j+1}}\right) \leq c_{i+1}\left(1-\rho^{-}+o(1)\right) .
$$

Hence $c_{k} \leq\left(1-\rho^{-}+o(1)\right)^{k-1} p$, provided $c_{k} \rightarrow \infty$. Summing we find that $\sigma(p)=\Sigma(\vec{c})=$ $p\left(1 / \rho^{-}+o(1)\right)$, provided $p \rightarrow \infty$. It follows from (8.1) that almost all compositions of $n$ contain $p$ provided $\log _{1 / r} n-p\left(1 / \rho^{-}+o(1)\right) \rightarrow \infty$. It suffices to have $p<\left(\rho^{-}-\delta\right) \log _{1 / r} n$ for any fixed $\delta>0$. Since we must have $(p+1) \in \mathcal{D}^{+}$, the largest possible value for $p$ may miss the upper bound by as much as a factor asymptotic to $\rho^{+}$. This gives the value $\rho^{+} \rho^{-}$appearing in Theorem 1 (g.ii).

To prove (g.i) for $D_{n}$, we cannot simply sum over the constructions in the previous paragraph because the number of elements in $\mathcal{D}^{+}$not exceeding some value $L$ may be as little as $\log _{1 / \rho^{+}} L$, which would lead to a lower bound for $D_{n}$ on the order of $\log \log n$. Instead, we remove the requirement that $p-1 \in \mathcal{D}^{+}$and build a mirror image construction $\vec{b}=\vec{\ell}\left(b_{s}\right), b_{s}, \ldots, b_{2}, b_{1}$ the same way we built $\vec{c}$, with $b_{1}=p$ and $b_{i}-b_{i+1}$ as large as possible. Summing we find that $\Sigma(\vec{b}) \leq p\left(1 / \rho^{+}-1+o(1)\right)$. We then use $\vec{d}=\vec{\ell}\left(b_{s}\right), b_{s}, \ldots, b_{2}, p, c_{2}, \ldots, c_{t}, \vec{r}\left(c_{t}\right)$, noting that $\Sigma(\overrightarrow{\vec{d}}) \leq C_{1} p$ for all $C_{1}>1 / \rho^{+}+1 / \rho^{-}-1$ and all sufficiently large $n$, depending on $C_{1}$. It suffices to take $\mathcal{P}(n)=\left\{k<C_{2} \log n\right\}$ where $C_{1} C_{2} \log (1 / r)<1$.

It remains to prove (g.iii). From (f) and (g.ii), it follows that $M_{n} \sim \log _{1 / r} n$ almost surely. Note that the conditions on $C_{1}$ and $C_{2}$ in the previous paragraph become $C_{1}>1$ and $C_{2}<1 / \log (1 / r)$. Thus $|\mathcal{P}(n)| \geq C_{3} \log _{1 / r} n$ for all $C_{3}<1$ and all sufficiently large $n$ depending on $C_{3}$. Since $D_{n} \leq M_{n}$, we are done by Theorem 1(e). 


\section{Average number of occurrences of a part}

One can use the generating function procedure from Section 3.5 to study the average number of parts of a given size $k$; however, there is an easier approach. Simply sum over $p$ the number of ways to form a composition of $p$ followed by a part of size $k$ followed by a composition of $(n-p-k)$, being careful to consider the empty compositions when $p=0$ or $n-p-k=0$. Then divide this result by the total number of compositions of $n$.

To illustrate, consider unrestricted compositions; that is, $\mathcal{L}=\mathcal{R}=\mathbb{N}$ and $\mathcal{D}=\mathbb{Z}$. Since there are $2^{n-1}$ compositions of $n$ when $n>0$, the average number of parts of size $k$ in a composition of $n$ is

$$
2^{1-n}\left(2^{n-k-1}+\sum_{p=1}^{n-k-1} 2^{p-1} 2^{n-p-k-1}+2^{n-k-1}\right)=2^{-k-1}(n-k+3)
$$

when $0<k<n$. Thus the average number of parts of size $k$ is asymptotic to $n 2^{-k-1}$, and so the average falls off by a factor of 2 each time the size is increased by 1 . The general behavior need not be this regular, but we do have the following formula.

Theorem 5 Suppose that either $\operatorname{gcd}\left(\mathcal{D}^{+} \cap \mathcal{D}^{-}\right)=1$ or $\operatorname{gcd}(\mathcal{D}-\mathcal{D})=1$ and let $r=r(\mathcal{D})$ be as in Theorem 1. We use the notation of (1.2) and Theorem 4. For fixed $k$, the average number of parts of size $k$ in a composition of $n$ is asymptotic to $B_{k} n r^{k-1}$ where

$$
B_{k}=\frac{z(r)\left(f\left(r, r, \mathcal{D}^{+}, \mathcal{R}_{k}^{\prime}\right)+\chi\left(1 \in \mathcal{R}_{k}\right)\right)\left(f\left(r, r, \mathcal{L}_{k}^{\prime}, \mathcal{D}^{-}\right)+\chi\left(1 \in \mathcal{L}_{k}\right)\right)}{\frac{d}{d r}\left(z(r) f\left(r, r, \mathcal{D}^{+}, \mathcal{D}^{-}\right)\right)},
$$

$\mathcal{R}_{k}=(\mathcal{D}-\{k\})^{-}$and $\mathcal{L}_{k}=(\mathcal{D}+\{k\})^{+}$.

Proof: We proceed as discussed above. For asymptotic purposes we can limit the index of summation $p$ to an interval $[\omega(1), n-\omega(1)]$. Use (3.2), noting that the coefficients of $F(x)+\frac{G(x)}{1-H(x)}$ are asymptotic to $\left(G(r) / r H^{\prime}(r)\right) r^{-n}$. A part $a$ immediately preceding the part of size $k$ must have $k-a \in \mathcal{D}$, which is equivalent to $-a \in(\mathcal{D}-\{k\})$. Likewise, for a following part $b, b-k \in \mathcal{D}$ and so $b \in(\mathcal{D}+\{k\})$.

Remark 6 By treating the part of size $k$ differently, we obtain alternate formulations of the preceding theorem. If we construct a composition up to and including the part followed by a composition without the part, we obtain the estimate

$$
\frac{z(r)\left(f\left(r, r, \mathcal{D}^{+},\{k-1\}\right)+\chi(k=1)\right)\left(f\left(r, r, \mathcal{L}_{k}^{\prime}, \mathcal{D}^{-}\right)+\chi\left(1 \in \mathcal{L}_{k}\right)\right)}{r \frac{d}{d r}\left(z(r) f\left(r, r, \mathcal{D}^{+}, \mathcal{D}^{-}\right)\right)} n
$$

for the average number of parts of size $k$. If the first composition ends with $k$ and the second begins with $k$ and we discard one of the extra copies of the part, we obtain

$$
\frac{z(r)\left(f\left(r, r, \mathcal{D}^{+},\{k-1\}\right)+\chi(k=1)\right)\left(f\left(r, r,\{k-1\}, \mathcal{D}^{-}\right)+\chi(k=1)\right)}{r^{k+1} \frac{d}{d r}\left(z(r) f\left(r, r, \mathcal{D}^{+}, \mathcal{D}^{-}\right)\right)} n .
$$


Example 4 (Carlitz compositions) For Carlitz compositions, the $B_{k}$ are asymptotically constant as $k \rightarrow \infty$ because $\mathcal{L}_{k}$ and $\mathcal{R}_{k}$ only exclude the part $k$. The asymptotic value is obtained by replacing $\mathcal{R}_{k}$ and $\mathcal{L}_{k}$ with $\mathbb{N}$, which is the same as $\mathcal{D}^{+}$and $\mathcal{D}^{-}$. However, there is a simpler formula in this case: Let $C(x)$ be the generating function for Carlitz compositions and $C_{k}(x)$ the generating function for Carlitz compositions with last part not equal to $k$, allowing the empty composition in the latter case. Then $1+C(x)=C_{k}(x)\left(1+x^{k}\right)$ and so $C_{k}(x)=\frac{C(x)+1}{1+x^{k}}$. The total number of parts of size $k$ in Carlitz compositions of $n$ is $\left[x^{n}\right]\left(C_{k}(x) x^{k} C_{k}(x)\right)$. Substituting the formula for $C_{k}(x)$, the average number of parts of size $k$ in a Carlitz composition of $n$ is

$$
\frac{\left[x^{n}\right]\left(x^{k} C(x)^{2} /\left(1+x^{k}\right)^{2}\right)}{\left[x^{n}\right] C(x)} \sim \frac{r^{k}\left[x^{n}\right]\left(C(x)^{2}\right)}{\left(1+r^{k}\right)^{2}\left[x^{n}\right] C(x)} \sim \frac{r^{k} n A^{2} r^{-n}}{\left(1+r^{k}\right)^{2} A r^{-n}}=n A \frac{r^{k}}{\left(1+r^{k}\right)^{2}},
$$

where $r<1$ is the radius of convergence of $C(x)$ and $A=\lim _{x \rightarrow r}((1-x / r) C(x))$, the value in Theorem 1(a). Summing the formula on $k$ provides an asymptotic formula for $\mu n$, the average number of parts. Thus $\mu=A \sum_{k \geq 1} \frac{r^{k}}{\left(1+r^{k}\right)^{2}}$ in the case of Carlitz compositions.

In contrast to Carlitz compositions, when $\mathcal{D}=\{ \pm 1,0\}$ we have $\mathcal{L}_{k}=\mathcal{R}_{k}=\{k \pm 1, k\}$ and so, by Theorem $1(\mathrm{~d}), B_{k}$ approaches zero as $k$ increases. Some values of $B_{k}$ are computed in the next section.

\section{Some computational results}

Call a subset $\mathcal{S}$ of $\mathbb{Z}^{+}$eventually periodic if there is a period $p$ and an integer $k$ such that, for $s \geq k, s \in \mathcal{S}$ if and only if $s+p \in \mathcal{S}$. (The sets $\mathcal{D}$ in Examples 1-3 are all eventually periodic.) If $\mathcal{D}$ is eventually periodic, only a finite number of the recursions (3.2) suffice to determine $f(x, x, \mathcal{D}, \mathcal{D})$. Thus, it is relatively straightforward to estimate $f(x, x, \mathcal{D}, \mathcal{D})$ for any given $x$ and so determine the radius of convergence. Since the right side of (3.2) is monotonic in the $f$ values, we can obtain upper and lower estimates for all computations by using two trivial bounds for $f\left(x, x^{\ell} t, \mathcal{L}, \mathcal{R}\right)$ for large $\ell$ : a lower bound of zero and an upper bound based on all compositions:

$$
0 \leq f\left(x, x^{\ell} t, \mathcal{L}, \mathcal{R}\right) \leq \sum\left(\begin{array}{l}
n-1 \\
k-1
\end{array}\right)\left(x^{\ell} t\right)^{k} x^{n}=\frac{x^{\ell} t}{1-\left(1+x^{\ell} t\right) x} .
$$

Since derivatives can also be computed and bounded, the asymptotic mean $n \mu$ and variance $n \sigma^{2}$ of the number of parts can be determined. Similarly, the constant $A$ in $c_{\mathcal{L}, \mathcal{R}}(n) \sim A r^{-n}$ can be determined when $\mathcal{L}$ and $\mathcal{R}$ are also eventually periodic. Results for various $\mathcal{D}$ and either $(\mathcal{L}, \mathcal{R})=\left(\mathcal{D}^{+}, \mathcal{D}^{-}\right)$or $\mathcal{L}=\mathcal{R}=\mathbb{N}$ are shown in Table 1 , except that we have not computed $\sigma$. Of course, the results for all compositions and Carlitz compositions were already known.

Remark 7 In [1] Andrews has considered compositions whose parts satisfy

$$
1 \leq c_{i+1} \leq c_{i}+d
$$




\begin{tabular}{|c|cccc|}
\hline $\mathcal{D}$ & $r(\mathcal{D})$ & $\mu(\mathcal{D})$ & $A\left(\mathcal{D}^{+}, \mathcal{D}, \mathcal{D}^{-}\right)$ & $A(\mathbb{N}, \mathcal{D}, \mathbb{N})$ \\
\hline $\mathbb{Z}$ & 0.50000000 & 0.50000000 & 0.50000000 & 0.50000000 \\
$\mathbb{Z} \backslash\{0\}$ & 0.57134979 & 0.35060127 & 0.45636347 & 0.45636347 \\
$\{0, \pm 1\}$ & 0.59944776 & 0.65837031 & 0.21325984 & 0.76243668 \\
$\{ \pm 1\}$ & 0.80821704 & 0.43952368 & 0.07733684 & 1.13479609 \\
odd & 0.67104361 & 0.31863928 & 0.19448851 & 0.64363806 \\
$\{0,-1\} \cup \mathbb{N}$ & 0.57614877 & 0.61491263 & 0.31236332 & 0.91805653 \\
$\{-1\} \cup \mathbb{N}$ & 0.73321632 & 0.42338859 & 0.17201062 & 2.14500651 \\
\hline
\end{tabular}

Table 1: Values of various constants in Theorem 1. The first two rows are the well-studied cases of unrestricted compositions and Carlitz compositions. The last two rows require that decreases in value be limited to steps of 1 . We have a central limit theorem in all cases but do not have a local limit theorems for $\mathcal{D}=\{ \pm 1\}$ or for $\mathcal{D}=$ odd.

In the case $d=1$, these correspond, via reversing the order of parts, to our case $\mathcal{D}=$ $\{0,1\} \cup \mathbb{N}$, which is the next to last example in Table 1. Andrews has determined the generating function

$$
1+\sum_{n \geq 1} c(n) x^{n}=\frac{1}{F(x)}
$$

with

$$
F(x)=\sum_{n=0}^{\infty} \frac{(-1)^{n} x^{n^{2}}}{(1-x)\left(1-x^{2}\right) \cdots\left(1-x^{n}\right)} .
$$

Thus, we have the numerical check

$$
F(0.57614877 \cdots) \stackrel{?}{=} 0,
$$

which holds to the accuracy with which we have computed the radius of convergence.

We have computed the exact value of $c_{\mathcal{L}, \mathcal{D}, \mathcal{R}}(n)$ for $n \leq 100$ in three cases:

$$
\begin{aligned}
& \mathcal{L}, \mathcal{D}, \mathcal{R}=\mathbb{N}, \mathbb{N}, \mathbb{N} ; \quad \text { (Carlitz compositions) } \\
& \mathcal{L}, \mathcal{D}, \mathcal{R}=\{1\},\{0, \pm 1\},\{1\} \\
& \mathcal{L}, \mathcal{D}, \mathcal{R}=\mathbb{N},\{-1\} \cup \mathbb{N}, \mathbb{N} .
\end{aligned}
$$

Table 2 compares the exact values to $A r^{-n}$ for $n=20,40,60,80,100$. For these three instances of $\mathcal{L}, \mathcal{D}, \mathcal{R}$ we have also computed the exact average number of $k$ 's for $1 \leq k \leq 5$ and $n \leq 100$. Tables 3 through 5 compare the exact averages to the estimate $B_{k} n r^{k-1}$ given by Theorem 5 . 


\begin{tabular}{|c|ccc|}
\hline$n$ & $\{0, \pm 1\}$ & $\{-1\} \cup \mathbb{N}$ & Carlitz \\
\hline 20 & 0.2133923072 & 1.976371127 & 0.45625300208 \\
40 & 0.2132602114 & 2.135882299 & 0.45636352310 \\
60 & 0.2132598395 & 2.144789362 & 0.45636347404 \\
80 & 0.2132598383 & 2.145003426 & 0.45636347406 \\
100 & 0.2132598382 & 2.145006149 & 0.45636347406 \\
\hline$A$ & 0.2132598387 & 2.145006511 & 0.4563634741 \\
\hline
\end{tabular}

Table 2: Entry $n$ is the total number of compositions of $n$ of the type indicated at the top of the column, divided by $r^{n}$. These ratios converge to $A$ as $n \rightarrow \infty$. In the first case, $\mathcal{L}=\mathcal{R}=\{1\}$ and in the other two cases $\mathcal{L}=\mathcal{R}=\mathbb{N}$.

\begin{tabular}{|c|ccccc|}
\hline$n$ & $k=1$ & $k=2$ & $k=3$ & $k=4$ & $k=5$ \\
\hline 20 & 0.1232064333 & 0.1673514703 & 0.1996036282 & 0.2170345124 & 0.2221236215 \\
40 & 0.1144045629 & 0.1577991873 & 0.1923920089 & 0.2149534345 & 0.2269324760 \\
60 & 0.1114699745 & 0.1545982152 & 0.1899986402 & 0.2142827963 & 0.2285128075 \\
80 & 0.1100026807 & 0.1529977353 & 0.1888019520 & 0.2139474717 & 0.2293029763 \\
100 & 0.1091223045 & 0.1520374474 & 0.1880839390 & 0.2137462769 & 0.2297770775 \\
\hline$B_{k}$ & 0.1056007996 & 0.1481962957 & 0.1852118872 & 0.2129414978 & 0.2316734825 \\
\hline
\end{tabular}

Table 3: Entry $(n, k)$ is the average number of $k$ 's in a Carlitz composition, divided by $\left(n r^{k-1}\right)$. These ratios converge to $B_{k}$ as $n \rightarrow \infty$.

\begin{tabular}{|c|ccccl|}
\hline$n$ & $k=1$ & $k=2$ & $k=3$ & $k=4$ & \multicolumn{1}{c|}{$k=5$} \\
\hline 20 & 0.4635323802 & 0.3982078306 & 0.0539727763 & 0.0010151669 & 0. \\
40 & 0.4097639407 & 0.4202779900 & 0.0772846537 & 0.0035140869 & 0.0000387011 \\
60 & 0.3917631945 & 0.4275355953 & 0.0851449533 & 0.0044514080 & 0.0000672187 \\
80 & 0.3827625231 & 0.4311640139 & 0.0890753265 & 0.0049205265 & 0.0000816553 \\
100 & 0.3773621192 & 0.4333410638 & 0.0914335510 & 0.0052019992 & 0.0000903181 \\
\hline$B_{k}$ & 0.3557605038 & 0.4420492631 & 0.1008664489 & 0.0063278902 & 0.0001249693 \\
\hline
\end{tabular}

Table 4: Here $\mathcal{L}, \mathcal{D}, \mathcal{R}=\{1\},\{0, \pm 1\},\{1\}$. Entry $(n, k)$ is the average number of $k$ 's in an $(\mathcal{L}, \mathcal{D}, \mathcal{R})$ composition, divided by $\left(n r^{k-1}\right)$. These ratios converge to $B_{k}$ as $n \rightarrow \infty$. 


\begin{tabular}{|c|ccccc|}
\hline$n$ & $k=1$ & $k=2$ & $k=3$ & $k=4$ & $k=5$ \\
\hline 20 & 0.09418367347 & 0.1704121644 & 0.1458658253 & 0.08426137726 & 0.0533116822 \\
40 & 0.09381642011 & 0.1812162742 & 0.1670051345 & 0.09815898620 & 0.0479774518 \\
60 & 0.09544927562 & 0.1897682933 & 0.1786950076 & 0.1045258792 & 0.0468131827 \\
80 & 0.09638926344 & 0.1943347881 & 0.1848809773 & 0.1079137715 & 0.0462271514 \\
100 & 0.09695980748 & 0.1970846924 & 0.1886044195 & 0.1099581659 & 0.0458762017 \\
\hline$B_{k}$ & 0.0992435949 & 0.2080874564 & 0.2035002652 & 0.1181365921 & 0.0444734586 \\
\hline
\end{tabular}

Table 5: Here $\mathcal{L}, \mathcal{D}, \mathcal{R}=\mathbb{N},\{-1\} \cup \mathbb{N}, \mathbb{N}$. Entry $(n, k)$ is the average number of $k$ 's in an $(\mathcal{L}, \mathcal{D}, \mathcal{R})$ composition, divided by $\left(n r^{k-1}\right)$. These ratios converge to $B_{k}$ as $n \rightarrow \infty$.

\section{References}

[1] G. E. Andrews, The Rogers-Ramanujan reciprocal and Minc's partition function, Pacific J. of Mathematics 95 (1981) 251-256.

[2] E.A. Bender and E.R. Canfield, Locally restricted compositions II: General restrictions, in preparation.

[3] E.A. Bender and L.B. Richmond, Central and local limit theorems applied to asymptotic enumeration II: Multivariate generating functions, J. Combin. Theory Ser. A 34 (1983) 255-265.

[4] L. Carlitz, Restricted compositions, Fibonacci Quart. 14 (1976) 254-264.

[5] W.M.Y. Goh and P. Hitczenko, Average number of distinct part sizes in a random Carlitz composition, European J. Combin. 23 (2002) 647-657.

[6] S. Heubach and T. Mansour, Counting rises, levels, and drops in compositions, preprint. Available at arXiv:math.CO/0310197.

[7] P. Hitczenko and G. Louchard, Distinctness of compositions of an integer: a probabilistic analysis, Random Structures Algorithms 19 (2001) 407-437.

[8] P. Hitczenko and G. Stengle, Expected number of distinct part sizes in a random integer composition, Combin. Probab. Comput. 9 (2000) 519-527.

[9] H.-K. Hwang and Y. Yeh, Measures of distinctness for random partitions and compositions of an integer, Adv. Appl. Math. 19 (1997) 378-414.

[10] A. Knopfmacher and H. Prodinger, On Carlitz compositions, European J. Combin. 19 (1998) 579-589.

[11] G. Louchard and H. Prodinger, Probabilistic analysis of Carlitz compositions, Discrete Math. Theor. Comput. Sci. 5 (2002) 71-96.

[12] L. Takács, Queueing methods in the theory of random graphs, Chapter 2 of Advances in Queueing. Theory, Methods, and Open Problems, Edited by Jewgeni H. Dshalalow, CRC Press, New York, 1995, pages 45-78. 